\title{
Novel tau fragments in cerebrospinal fluid: relation to tangle pathology and cognitive decline in Alzheimer's disease
}

\author{
Claudia Cicognola ${ }^{1}$. Gunnar Brinkmalm ${ }^{1}$. Jessica Wahlgren ${ }^{1}$. Erik Portelius ${ }^{1,2}$. Johan Gobom ${ }^{1,2}$. \\ Nicholas C. Cullen ${ }^{3} \cdot$ Oskar Hansson ${ }^{4,5} \cdot$ Lucilla Parnetti $^{6} \cdot$ Radu Constantinescu $^{7} \cdot$ Kristin Wildsmith $^{8}$. \\ Hsu-Hsin Chen ${ }^{9} \cdot$ Thomas G. Beach $^{10}$ - Tammaryn Lashley ${ }^{11} \cdot$ Henrik Zetterberg ${ }^{1,2,12,13}$. Kaj Blennow ${ }^{1,2}$. \\ Kina Höglund ${ }^{1,2,14}$
}

Received: 22 October 2018 / Revised: 10 December 2018 / Accepted: 10 December 2018 / Published online: 13 December 2018 (c) The Author(s) 2018

\begin{abstract}
Tau is an axonal microtubule-binding protein. Tau pathology in brain and increased tau concentration in the cerebrospinal fluid (CSF) are hallmarks of Alzheimer's disease (AD). Most of tau in CSF is present as fragments. We immunoprecipitated tau from CSF and identified several endogenous peptides ending at amino acid (aa) 123 or 224 using high-resolution mass spectrometry. We raised neo-epitope-specific antibodies against tau fragments specifically ending at aa 123 and 224, respectively. With these antibodies, we performed immunohistochemistry on brain tissue and designed immunoassays measuring $\mathrm{N}-123, \mathrm{~N}-224$, and $\mathrm{x}-224$ tau. Immunoassays were applied to soluble brain fractions from pathologically confirmed subjects (81 AD patients, 33 controls), CSF from three cross-sectional and two longitudinal cohorts (a total of $133 \mathrm{AD}, 38 \mathrm{MCI}, 20$ MCI-AD, 31 PSP, 15 CBS patients, and 91 controls), and neuronally- and peripherally-derived extracellular vesicles (NDEVs and PDEVs, respectively) in serum from four AD patients and four controls. Anti-tau 224 antibody stained neurofibrillary tangles and neuropil threads, while anti-tau 123 only showed weak cytoplasmic staining in AD. N-224 tau was lower in the AD soluble brain fraction compared to controls, while N-123 tau showed similar levels. N-224 tau was higher in AD compared to controls in all CSF cohorts $(p<0.001)$, but not N-123 tau. Decrease in cognitive performance and conversion from MCI to AD were associated with increased baseline CSF levels of N-224 tau $(p<0.0001)$. N-224 tau concentrations in PSP and CBS were significantly lower than in $\mathrm{AD}(p<0.0001)$ and did not correlate to t-tau and p-tau. In a longitudinal cohort, CSF N-224 tau levels were stable over 6 months, with no significant effect of treatment with AChE inhibitors. N-224 tau was present in NDEVs, while N-123 tau showed comparable concentrations in both vesicle types. We suggest that N-123 tau is produced both in CNS and PNS and represents a general marker of tau metabolism, while N-224 tau is neuron-specific, present in the tangles, secreted in CSF, and upregulated in $\mathrm{AD}$, suggesting a link between tau cleavage and propagation, tangle pathology, and cognitive decline.
\end{abstract}

Keywords Alzheimer's disease $\cdot$ Tau fragments $\cdot$ Cerebrospinal fluid $\cdot$ Mass spectrometry $\cdot$ Immunohistochemistry

\section{Introduction}

Alzheimer's disease (AD) is the most common neurodegenerative tauopathy and is responsible for $75 \%$ of the 35.6 million dementia cases worldwide [44]. As the world population

Electronic supplementary material The online version of this article (https://doi.org/10.1007/s00401-018-1948-2) contains supplementary material, which is available to authorized users.

Claudia Cicognola

claudia.cicognola@neuro.gu.se

Extended author information available on the last page of the article ages, the frequency is expected to double by 2030 and triple by 2050 [44]. At present, the only therapeutic option is symptomatic, while disease-modifying therapies are still under development [37].

The classical pathological hallmarks of AD consist of deposition of amyloid beta (A $\beta)$ in cortical plaques and hyperphosphorylation of tau and formation of neurofibrillary tangles (NFT) causing neuronal degeneration [37]. In cerebrospinal fluid (CSF), these pathological changes are reflected by low $\mathrm{A} \beta 42$ levels, low $\mathrm{A} \beta 42 / \mathrm{A} \beta 40$ ratio, and high levels of phosphorylated tau (p-tau) and total tau (t-tau) $[12,32]$. Tau pathology, namely the aggregation of tau and 
formation of tangles, is described as a mechanism common to AD and primary tauopathies, such as corticobasal neurodegeneration (CBD) and progressive supranuclear palsy (PSP). Tau pathology is predominantly localized to neurons in $\mathrm{AD}$, while in primary tauopathies is also present in the glia in filamentous (tufted astrocytes, in PSP) or more diffuse form (astrocytic plaques, in CBD). Their clinical presentation is very different, with $\mathrm{AD}$ being primarily characterized by memory impairment and PSP by parkinsonism; CBD has a very heterogeneous clinical presentation, ranging from parkinsonism to AD-like symptoms, which goes under the name of corticobasal syndrome (CBS) [7]. The use of $\mathrm{AD}$ biomarkers to better differentiate $\mathrm{AD}$-like $\mathrm{CBS}$ has been proposed to help diversify the therapeutic options [15]. Nonetheless, several studies on CSF in primary tauopathies show normal or even low concentrations of t-tau and p-tau, in stark contrast to the increased levels seen in $\mathrm{AD}$, and no CSF profile specific for primary tauopathies has been established [15, 21, 41, 42].

From a methodological perspective, most of the data on CSF t-tau come from studies based on commercially available immunoassays that rely on a combination of monoclonal antibodies (AT120, HT7, and BT2) having epitopes in the mid-region of the protein (Fig. 1a), but several studies suggest that tau is present as different fragments in both brain extract and CSF $[3,9,10,22,30,35,38]$. A recent study used high-performance liquid chromatography (HPLC) separation of CSF proteins, followed by Western blotting with tau antibodies binding to different tau domains, to show that CSF tau is composed of a series of fragments, with N-terminal and mid-region tau representing the most abundant variants in CSF [30]. Mass spectrometry (MS) has also proven helpful to characterize tau species in detail. However, CSF is a complex matrix and several pre-treatments had to be tested to reach the adequate sensitivity to detect tau and its variants in CSF [9, 10, 35]. Different groups have detected tau peptides spanning from the $\mathrm{N}$ - to the C-terminus of the protein in CSF $[9,10,35]$. However, the most reproducible result so far is increased concentrations of C-terminally truncated tau forms in $\mathrm{AD}$, which fits well with the highly reproducible results that have been generated using regular t-tau and p-tau immunoassays [32], whilst the other neurodegenerative dementias, including primary tauopathies, are surprisingly normal $[9,10]$.

A possible explanation for the AD specificity of CSF tau profiles has been proposed, based on an in vivo model using stable-isotope labeling on human central nervous system (CNS) and iPSC-derived neurons [36]. The results show that most tau in CSF and cell media lack the microtubule-binding region and more $\mathrm{C}$-terminal parts, that these tau forms are actively secreted from neurons, and that the secretion appears to be stimulated by A $\beta$ exposure [36]. CSF tau concentrations generated using the currently available assays would thus not be specific for tangle pathology or neurodegeneration but rather reflect a neuronal response to $\mathrm{A} \beta$ pathology.

To identify novel tau biomarkers that might better reflect AD-type neurodegeneration, we used N-terminal and midregion-specific antibodies for enrichment of tau species followed by MS analysis using both bottom-up and top-down approaches. We identified a large number of endogenous and tryptic peptides belonging to two different pools of tau,
Fig. 1 Overview of standard (a) and novel $(\mathbf{b}, \mathbf{c})$ immunoassays for t-tau and tau fragments: the traditional assay only detects the mid-region of tau; our novel immunoassays detect fragments ending at aa123 and aa224
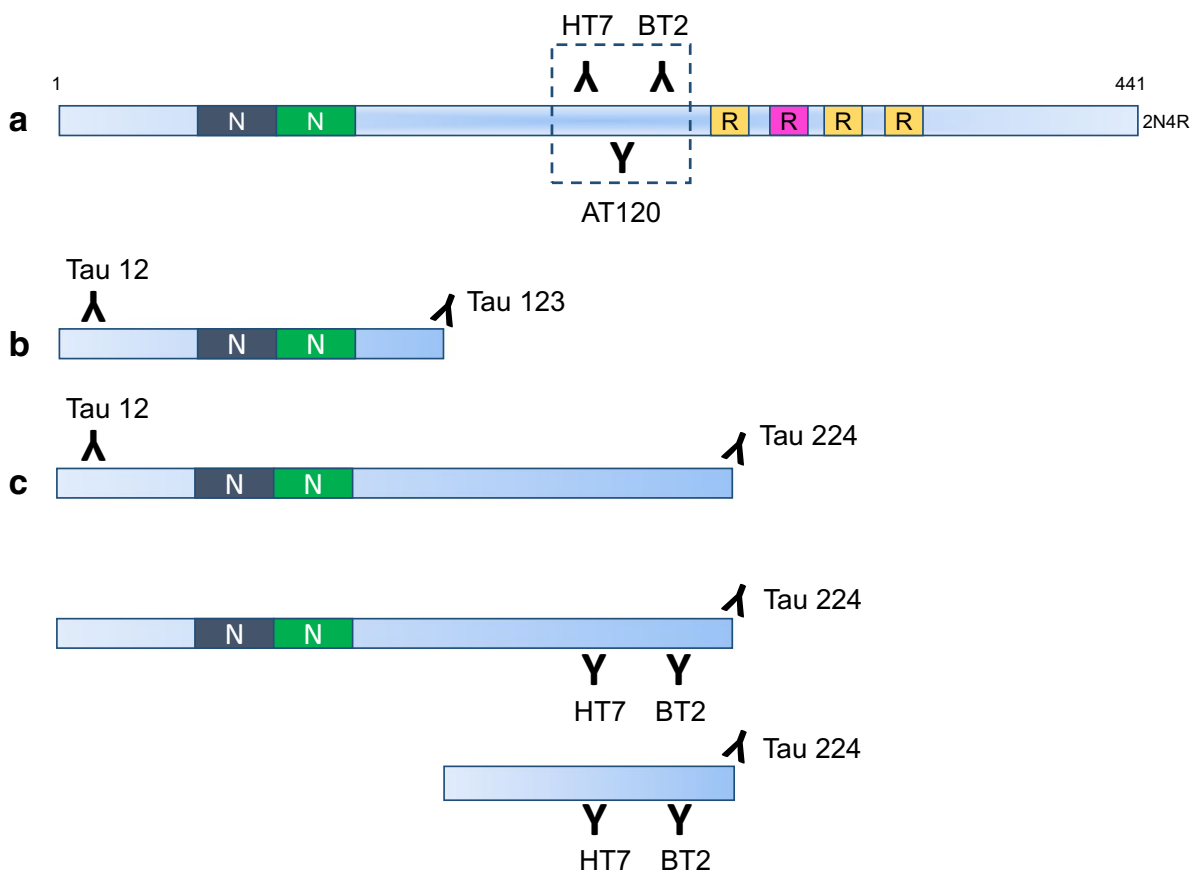
one starting at the N-terminal and ending at aa 123 through 129 and another mid-region pool covering aa 144-224 (tau 441 numbering). We then immunized mice to generate neo-epitope-specific antibodies against aa 123 and 224. We applied these unique antibodies for further studies evaluating the link between these specific tau fragments and tau pathology. We immunohistochemically stained post-mortem brain and developed immunoassays to specifically quantify the respective fragment in soluble brain extracts and CSF from AD, PSP, CBS patients, and controls. We also evaluated the N-224 fragment stability as biomarker in a longitudinal cohort under treatment with acetylcholinesterase (AChE) inhibitors. Furthermore, we analyzed the two fragments in neuronally derived extracellular vesicles (NDEV), including exosomes, isolated from serum, to study the potential role of exosomes in tau propagation. The present study aimed to characterize new tau fragments and describe a possible model for their distribution, function, and role in the pathogenesis of $\mathrm{AD}$.

\section{Materials and methods}

\section{Brain tissue extraction for immunoprecipitation}

Brain tissue for the immunoprecipitation mass spectrometry (IP-MS) analysis was from the Netherlands Brain Bank. Samples were collected from the superior parietal lobe of control brains (Braak 0-I) 4-7 h post-mortem and stored at $-80{ }^{\circ} \mathrm{C}$. Brain material was kept on dry ice and $100 \mathrm{mg}$ samples were excised and put in TBS solution (20 mM Tris, $137 \mathrm{mM} \mathrm{NaCl}$, and $\mathrm{pH}$ 7.6) containing protease inhibitor (Protease Inhibitor Cocktail tablets, Roche, Cat no: 11697 498 001, Lot nr: 14,251,300) and homogenized with a motor homogenizer to final a tissue:TBS ratio of 1:5. The tissue homogenates were further diluted 1:2 in TBS solution with protease inhibitor and vortexed. The samples were then centrifuged for $60 \mathrm{~min}$ at $31,000 \times g,+4{ }^{\circ} \mathrm{C}$. Supernatants were collected, aliquoted, and stored at $-80{ }^{\circ} \mathrm{C}$ pending analysis. Aliquots were further diluted 1:40 in TBS prior to IP.

\section{Immunoprecipitation}

Aliquots (4 $\mu \mathrm{g}$ ) of Tau 12 (binding region aa 9-18, Nordic Biosite), HT7 (aa 159-163, Thermo Scientific), and BT2 (aa 194-198, Thermo Scientific) were added to $50 \mu \mathrm{L} /$ each magnetic Dynabeads M-280 and incubated $2 \mathrm{~h}$ on a rocking platform at room temperature. The beads were washed three times with double volume of PBS (10 mM Na-phosphate, $0.15 \mathrm{M} \mathrm{NaCl}$, and $\mathrm{pH} 7.4)$. The antibody was crosslinked using $20 \mathrm{mM}$ dimethyl pimelimidate dihydrochloride (DMP; Sigma-Aldrich) and 0.2 M triethanolamine ( $\mathrm{pH} 8.2$, Sigma-Aldrich) according to the manufacturer's product description. The cross-linked beads were washed twice in PBS and blocked with Roti-Block (Carl Roth) for $1 \mathrm{~h}$ on a rocking platform at room temperature. Antibody-conjugated beads and Tween 20 (final concentration in the sample: $0.025 \%$ ) were added to $3 \mathrm{~mL} \mathrm{CSF}$ or $7 \mu \mathrm{L}$ of a TBS-soluble fraction of brain homogenate diluted in $270 \mu \mathrm{L}$ PBS. For some of the samples intended for tryptic digestion, $100 \mathrm{fmol}$ of a $13 \mathrm{C} 15 \mathrm{~N}$ lysine- and arginine-labeled tau 1N4R protein standard (provided by Dr. Thomas McAvoy, Merck Research Laboratories) were added. Samples were incubated overnight on a rocking platform at $+4{ }^{\circ} \mathrm{C}$. The magnetic beads/ sample solution was transferred to a magnetic particle processor (KingFisher, Thermo Fisher Scientific) (tube 1). The following three wash steps (tubes 2-4) were conducted for $10 \mathrm{~s}$ in $1 \mathrm{~mL}$ of each washing buffer: $0.025 \%$ Tween 20 in PBS (tube 2), PBS (tube3), and $50 \mathrm{mM}$ ammonium hydrogen carbonate (tube $4, \mathrm{pH} 8.0$ ). Tau was eluted from the beads by adding $100 \mu \mathrm{L} 0.5 \%$ formic acid (tube 5) for $4 \mathrm{~min}$. The eluted fractions were transferred to $0.65 \mathrm{~mL}$ prelubricated microcentrifuge tubes (Costar, Cat. 3206) and dried in a vacuum centrifuge. After drying, $10 \mu \mathrm{L}$ of a solution of $1 \mu \mathrm{g}$ trypsin in $390 \mu \mathrm{L} 50 \mathrm{mM}$ ammonium hydrogen carbonate was added to half of the samples and incubated overnight at $+37{ }^{\circ} \mathrm{C}$. The reaction was stopped by adding $2 \mu \mathrm{L}$ of $10 \%$ formic acid in ultra-pure water. The other half was analyzed intact for endogenous peptides. Samples were dried in a vacuum centrifuge and stored at $-80{ }^{\circ} \mathrm{C}$ pending analysis. Following the same protocol described above, we immunoprecipitated tau from CSF and brain lysate with the neo-epitope-specific antibodies to verify their specificity.

\section{LC-MS/MS analysis}

NanoLC coupled to ESI high-resolution hybrid quadrupoleorbitrap MS was performed with a Dionex 3000 system and either a Q Exactive or an Orbitrap Fusion (all three from Thermo Fisher Scientific, Inc.) in a similar way as previously published [15]. Immunoprecipitated samples were reconstituted in $7 \mu \mathrm{L} 8 \%$ formic acid/8\% acetonitrile in water. Samples $(6 \mu \mathrm{l})$ were loaded onto an Acclaim PepMap C18 trap column (length $20 \mathrm{~mm}$, internal diameter $75 \mu \mathrm{m}$, particle size $3 \mu \mathrm{m}$, pore size $100 \AA$, Thermo Fisher Scientific, Inc.) for desalting and sample clean-up. Sample loading buffer was $0.05 \%$ trifluoroacetic acid in water. Separation was performed by reversed-phase Acclaim PepMap C18 analytical columns (lengths 150 or $500 \mathrm{~mm}$, internal diameter $75 \mu \mathrm{m}$, particle size $2 \mu \mathrm{m}$, pore size $100 \AA$, and Thermo Fisher Scientific, Inc.). Separation was performed at a flow rate of 300 or $150 \mathrm{~nL} / \mathrm{min}$ by applying a 50-min-long linear gradient from 3 to $40 \% \mathrm{~B}$. Buffer A was $0.1 \%$ formic acid in water and buffer B was $0.1 \%$ formic acid $/ 84 \%$ acetonitrile in water. The mass spectrometer was set to operate in data-dependent mode using higher energy collision-induced 
dissociation (HCD) for ion fragmentation. For endogenous peptides, the acquisition parameters were optimized for large fragments. Acquisition settings were the same for MS and MS/MS acquisitions for a better quality MS/MS data: resolution setting 70,000, 1 microscan, target values 106, and trap injection time $250 \mathrm{~ms}$. For trypsinated samples, the MS/MS resolution setting was 17,500 and the trap injection time was $60 \mathrm{~ms}$.

\section{Processing of LC-MS/MS data}

LC-MS/MS acquisitions of tryptic digests were processed directly using Proteome Discoverer v1.4 (Thermo Fisher Scientific, Inc.) and Mascot database search software v2.6.1 (Matrix Science). Searches were made against both Uniprot and a custom made tau-only database. Acquisitions of non-digested samples were processed using Mascot Daemon v2.6/Mascot Distiller v2.6.3 (both Matrix Science) for charge and isotope deconvolution before submitting searches using Mascot. The same databases as for the digested samples were utilized. Processing and search settings are described in [17] and supplementary information.

\section{Expression of recombinant Tau 123 and 224}

Tau 123 (aa 1-123 of Tau F) and Tau 224 (aa 1-224) were PCR amplified using primers containing 5' Sgf I and 3' Not I sites with cDNA for Tau F (RC213312, Origene) as template. The PCR fragments were purified and cloned into Sgf I/Not I digested pEX_N_His_GST expression plasmid (PS100028, OriGene Technologies). 5' primer sequence was TTCTAAGCGATCGCCATGGCTGAGCCCC, 3' primer sequence for the 123 fragment was TAAAGCGGC CGCTTAGGTCACGTGACCAGCAGCTT, and 3' primer sequence for the 224 fragment was TAAAGCGGCCGCTTA CTTGGGCTCCCGGGTGGGT. Constructs were sequenced and transfected into E. coli BL21 (DE3). E. coli BL21 (DE3) containing the different constructs was incubated overnight in $50 \mathrm{~mL} \mathrm{LB}$ medium with ampicillin at a concentration of $100 \mu \mathrm{g} / \mathrm{mL}$. The overnight culture was used to inoculate $1 \mathrm{~L}$ of LB media with ampicillin $(100 \mu \mathrm{g} / \mathrm{mL})$, and when OD600 reached $0.4-0.6$, protein expression was induced with $0.5 \mathrm{mM}$ IPTG for $4 \mathrm{~h}$ at $+37^{\circ} \mathrm{C}$. The culture was centrifuged at $5500 \times g$ for $20 \mathrm{~min}$ at $+4{ }^{\circ} \mathrm{C}$ and the dry weight was calculated. The pellet was stored at $-20{ }^{\circ} \mathrm{C}$ pending purification. The pellet was resuspended in $5 \mathrm{~mL} / \mathrm{mg}$ of lysis buffer (20 mM Tris, $150 \mathrm{mM} \mathrm{NaCl}$, and 1\% NP40 pH 7.5) plus complete protease inhibitor (Roche) and incubated with rotation for $30 \mathrm{~min}$ at room temperature, after which the lysate was centrifuged at $17,000 \times g$ for $20 \mathrm{~min}$ at $+4{ }^{\circ} \mathrm{C}$ and the supernatant was collected. Protein extract was added to $50 \%$ Glutathione-Sepharose 4b (GE Healthcare) equilibrated with PBS and incubated for 30 min with rotation at room temperature. Sepharose was washed with PBS and the GST-Tau fusion protein was incubated with elution buffer (100 mM Tris- $\mathrm{HCl}$ and $120 \mathrm{mM} \mathrm{NaCl} \mathrm{pH} \mathrm{8.0,} \mathrm{with} 20 \mathrm{mM}$ glutathione) for $10 \mathrm{~min}$. The unbound fusion protein was eluted and the incubation step was repeated four times. Thrombin cleavage of the GST-fusion protein bound to the GST Sepharose was done in PBS with $50 \mathrm{U}$ thrombin for two and a half hours at room temperature. The cleaved, untagged protein was eluted with PBS containing protease inhibitor.

\section{Generation of monoclonal antibodies}

Monoclonal antibodies against the recombinant protein fragments were generated by immunization of 8-weekold Balb/c mice with KLH-conjugated peptides (Caslo) in complete Freund's adjuvant (Sigma). Peptide sequence was (KLH)-CEEAGIGDTPSLEDEAAGHVT for the 123 and (KLH)-CGGGRTPSLPTPPTREPK for the 224. After 2-3 dosages with the recombinant protein fragment (approximately $75 \mu \mathrm{g} /$ mouse), the spleen was removed and B cells were fused with the myeloma cell line SP2/0 following the standard procedures. Approximately 10 days after fusion, cell media were screened for the antibodies using full-length recombinant tau and recombinant protein fragments. Clones that reacted with the recombinant protein fragments but not with full-length tau and negative control protein were further grown, subcloned, and subsequently frozen in liquid nitrogen. The isotype was determined using a commercially available kit (Pierce Rapid Isotyping Kit-Mouse). Finally, antibodies were purified using a protein $\mathrm{G}$ column (GE healthcare).

\section{Immunohistochemistry}

Immunohistochemical analysis of the tau antibodies was carried out on brains that were donated to the Queen Square Brain Bank for Neurological Disorders, UCL Institute of Neurology, University College London. Seven-micron-thick formalin-fixed paraffin-embedded tissue sections were cut from the frontal cortex of pathologically diagnosed AD cases and neurologically normal controls. Tau immunohistochemistry required pressure cooker pre-treatment in citrate buffer $\mathrm{pH}$ 6.0. Endogenous peroxidase activity was blocked with $0.3 \% \mathrm{H} 202$ in methanol and non-specific binding with $10 \%$ dried milk solution. Tissue sections were incubated with the primary anti-tau 123 , anti-tau 224 , and AT8 (binding to Ser202 and Thr205) antibodies for $1 \mathrm{~h}$ at room temperature, followed by biotinylated anti-mouse $\operatorname{IgG}(1: 200$, $30 \mathrm{~min}$; DAKO) and ABC complex (30 min; DAKO). Colour was developed with di-aminobenzidine $/ \mathrm{H}_{2} \mathrm{O}_{2}$ [26]. Sections were viewed and imaged on a Nikon Eclipse. 


\section{ELISA assay for Tau N-123}

The immunoassay $\mathrm{N}-123$, detecting fragments going from the N-terminal (antibody-binding region: aa 9-18) to aa 123, was developed on an ELISA platform (Fig. 1b). 96-well plates were coated and incubated overnight at $+4{ }^{\circ} \mathrm{C}$ with in house antibody anti-tau 123 at a concentration of $6 \mu \mathrm{g} /$ $\mathrm{mL}$ in carbonate buffer ( $\mathrm{pH}$ 9.6). After blocking and washing, $90 \mu \mathrm{L}$ of titrated calibrator (123 recombinant tau fragment) and sample were co-incubated with $10 \mu \mathrm{L}$ of biotinylated detection antibody Tau 12 (Nordic Biosite), at a final concentration of $0.617 \mu \mathrm{g} / \mathrm{mL}$. CSF samples were run neat, brain tissue extracts were run at 50 -fold dilution. After overnight incubation at $+4{ }^{\circ} \mathrm{C}$, the plate was washed and incubated for $30 \mathrm{~min}$ with a streptavidin-HRP-conjugated solution (KemEnTek Diagnostics). After a final wash and addition of the chromogen tetramethylbenzidine (TMB ONE, KemEnTek Diagnostics), the plate was read on a Magellan reader at a wavelength of $450 \mathrm{~nm}$.

\section{Simoa assay for tau N-224}

The immunoassay (N-224) detecting fragments going from the N-terminal (antibody-binding region: aa 9-18) to aa 224 was developed using single-molecule array (Simoa, Quanterix, Lexington, MA, USA) technology (Fig. 1c). Magnetic beads (Quanterix, Lexington, MA, USA) were conjugated with the capture antibody anti-tau 224 at $0.3 \mathrm{mg} / \mathrm{mL}$ according to bead supplier's conjugation protocol. Prior to each run, Tau 224 recombinant protein calibrator was serially diluted and the biotin-labeled detection antibody Tau 12 (Nordic Biosite) was diluted to $0.5 \mu \mathrm{g} / \mathrm{mL}$ in PBS-Tween with $1 \%$ BSA. For each determination, 140,000 assay beads were washed and resuspended in $100 \mu \mathrm{L}$ of $\mathrm{CSF} / \mathrm{brain}$ extract, quality control sample, or calibrator. After a washing step, $20 \mu \mathrm{L}$ of detection antibody was added followed by 30 -min incubation. After a final wash, beads were resuspended in $100 \mu \mathrm{L}$ streptavidin-conjugated $\beta$-galactosidase (SBG, Quanterix, Lexington, MA, USA) at $150 \mathrm{pM}$ diluted in SBG Diluent (Quanterix, Lexington, MA, USA). Following $5 \mathrm{~min}$ of incubation, the beads were washed and transferred together with resorufin-D-galactopyranoside substrate (RGP, Quanterix, Lexington, MA, USA) to an array of wells, each well only big enough to contain one bead. The array was imaged with a charge-coupled device (CCD) camera imaging system and the images were used to differentiate between empty beads and beads with bound analyte, giving a signal expressed as average enzyme per bead (AEB). To extract concentrations from AEBs, each sample AEB was fitted to a four-parameter logistic curve plotted from the known concentrations of the Tau 224 calibrator run in parallel with the samples. Calibrator points were run in triplicates, while samples were run in duplicates. CSF samples were run neat and brain tissue extracts were run at 100,000-fold dilution.

\section{ELISA assay for Tau x-224}

A second immunoassay (x-224) detecting N-terminally truncated fragments ending at aa 224 (antibody-binding region: aa 159-198) was developed on an ELISA platform (Fig. 1c). 96-well plates were coated and incubated overnight at $+4{ }^{\circ} \mathrm{C}$ within house antibody anti-tau 224 at a concentration of $2 \mu \mathrm{g} / \mathrm{mL}$. After blocking and washing, $75 \mu \mathrm{L}$ of titrated calibrator (224 recombinant tau fragment) and neat CSF sample were co-incubated with $12.5 \mu \mathrm{L}$ of mixed biotinylated detection antibody HT7 and BT2 (Thermo Scientific), at a final concentration of $0.0625 \mu \mathrm{g} / \mathrm{mL}$. After overnight incubation at $+4{ }^{\circ} \mathrm{C}$, the plate was washed and incubated for $30 \mathrm{~min}$ with a streptavidin-HRP-conjugated solution (KemEnTek Diagnostics). After a final wash and addition of the chromogen tetramethylbenzidine (TMB ONE, KemEnTek Diagnostics), the plate was read on a Magellan reader at a wavelength of $450 \mathrm{~nm}$.

\section{Brain tissue samples}

All brain lysates were made from cryosectioned fusiform gyrus from $81 \mathrm{AD}$ and 33 controls at Banner Sun Health Institute and classified by Braak stages [16]. This region was chosen due to its close proximity to the entorhinal cortex and higher densities of NFTs than any other neocortical region. Subjects were consented, enrolled and annually assessed in the Arizona Study of Aging and Neurodegenerative Disorders, and then autopsied in the Brain and Body Donation Program [11]. The protocol and consent are annually reviewed by an Institutional Review Board. Brain tissue was collected $5 \mathrm{~h}$ or less after death and stored until used at $-80^{\circ} \mathrm{C}$. The cryosections were lysed in RIPA buffer with protease inhibitors (Protease Inhibitor Cocktail tablets, Roche) using the Qiagen TissueLyzer to a final concentration of $2-3 \mathrm{mg} / \mathrm{ml}$, as measured with BCA Protein Assay kit (Pierce). Samples were further diluted to 1:50 for the N-123 and t-tau measurements and to $1: 100,000$ for the $\mathrm{N}-224$ measurement in their respective assay buffer-containing protease inhibitor (Protease Inhibitor Cocktail tablets, Roche).

\section{CSF collection and biomarker analyses}

All CSF samples were collected by lumbar puncture in the L3/L4 or the L4/L5 inter-space, in the morning. The first $12 \mathrm{~mL}$ of CSF were collected in a polypropylene tube and immediately transported to the local laboratory and centrifuged $\left(10 \mathrm{~min}\right.$ at $1800 \mathrm{~g}$ at $\left.+20{ }^{\circ} \mathrm{C}\right)$. The supernatant was gently mixed to avoid possible gradient effects, aliquoted in polypropylene tubes, and stored at $-80{ }^{\circ} \mathrm{C}$. CSF t-tau 
and p-tau concentrations were measured using ELISA (INNOTEST ${ }^{\circledR}$ htau Ag and PHOSPHO_TAU (181P), Fujirebio) as previously described [13, 43]. CSF A $\beta 1-42$ was measured using an ELISA (INNOTEST ${ }^{\circledR} \mathrm{A} \beta 1-42$ ), specifically constructed to measure $\mathrm{A} \beta$ starting at amino acid 1 and ending at amino acid 42 [6].

\section{Discovery CSF cohort}

Non-AD subjects $(n=20)$ were patients referred to the hospital for the evaluation of psychiatric or neurological symptoms, but with basic (cell count, albumin ratio, and IgG index) and core (A $\beta 1-42$, t-tau, and p-tau) CSF biomarkers within normal ranges. Patients with $\mathrm{AD}(n=20)$ had a clinical diagnosis of AD along with biomarker positive CSF (Supplementary Table 4a). Samples were selected based on biomarker cut-offs for A $\beta 1-42$, t-tau, and p-tau 181 . Cut-offs used to for a biomarker positive profile was $A \beta 1$ $42<550 \mathrm{ng} / \mathrm{L}, \mathrm{t}$-tau $>400 \mathrm{ng} / \mathrm{L}$, and $\mathrm{p}$-tau $>80 \mathrm{ng} / \mathrm{L}$. The CSF sample aliquots used were de-identified left-over aliquots from clinical routine analyses, following a procedure approved by the Ethics Committee at University of Gothenburg (EPN 140,811).

\section{Validation CSF cohort}

The CSF sample aliquots used were from the Memory Clinic at the University of Lund (Dnr 695/2008). Included participants were characterized as $\mathrm{AD}(n=46)$, according to the criteria of National Institute of Neurological and Communicative Disorders and Stroke and the AD and Related Disorders Association [19, 28], and healthy volunteers as the control group $(n=50)$ (Supplementary Table $4 b)$. The applied IWG- 2 criterion for allocation to the $\mathrm{AD}$ group was a low concentration of $\mathrm{A} \beta 1-42(<550 \mathrm{ng} / \mathrm{L})$ with a high level of t-tau (>400 ng/L) or p-tau181 (>80 ng/L).

\section{Longitudinal CSF cohort}

Baseline CSF samples from 16 AD, 38 MCI, 20 MCI-AD, and 21 other neurological diseases (OND) subjects were collected at the Center for Memory Disturbances of the University of Perugia (Supplementary Table 5). All patients underwent a baseline clinical examination with a complete neuropsychological assessment and blood chemistry, brain CT, and/or MRI scan to exclude the other causes of cognitive deficit. Subjects were clinically diagnosed according to the NIA-AA AD criteria [28] and Petersen's MCI criteria [33], with AD CSF biomarkers (A $\beta 1-42$, t-tau, and p-tau) used as support. Cognitive assessment at follow-up was performed through MMSE every 6 months for $\sim 2.3$ years. OND subjects had no clinical or neuropsychological evidence of cognitive impairment and were diagnosed with Parkinson's disease $(n=15)$, psychiatric disorder $(n=1)$, white-matter lesions $(n=2)$, Miller-Fisher syndrome $(n=1)$, epilepsy $(n=1)$, and paraneoplastic syndrome $(n=1)$. The study was conducted according to the Helsinki Declaration and approved by the local ethics committee (Prot. N. 19369/08/ $\mathrm{AV})$.

\section{PSP and CBS CSF cohort}

Patients with parkinsonism were diagnosed at the Department of Neurology, Movement Disorders Unit, Sahlgrenska University Hospital, Gothenburg, Sweden, by movement disorders specialists. Thirty-one subjects were diagnosed with probable or definite PSP according to the criteria from the National Institute of Neurological Disorders and Stroke and Society for Progressive Supranuclear Palsy (NINDSSPSP) [27]. Fifteen subjects were diagnosed with probable CBS according to the criteria from Armstrong et al. [7]. All patients underwent lumbar puncture as part of the diagnostic work-up. CSF biomarkers A $\beta 1-42$, t-tau, and p-tau were analyzed at the Clinical Neurochemistry Laboratory, Sahlgrenska University Hospital, Mölndal, Sweden (Supplementary Table 6). Based on the concentrations of $A \beta 1-42$, subjects were further divided in $A \beta$ positive $(A \beta+)$ and $A \beta$ negative $(\mathrm{A} \beta-)$. The cut-off applied for an $A \beta+$ profile was the same as the discovery and validation cohorts $(A \beta<550 \mathrm{ng} / \mathrm{L})$. A $\beta$ subjects were 15 in the PSP and seven in the CBS group. Concentrations of N-123 and N-224 were compared to the ones in the $\mathrm{AD}$ and control groups of the validation cohort; the ratio between QC samples from the different runs was used for normalization. The study was conducted according to the Helsinki Declaration and the collection of samples was approved by the Regional Ethical Board at the University of Gothenburg (n. 460-13, 462-95, and 210-95).

\section{Biomarker stability study CSF cohort}

CSF samples were collected during a 6-month multicenter open study on 51 patients with $\mathrm{AD}$ on continuous treatment with acetylcholine esterase (AChE) inhibitors, as previously described (Supplementary Table 7) [14, 23]. Briefly, all patients underwent a thorough clinical investigation which included medical history, physical, neurological, and psychiatric examinations. AD was diagnosed following NINCDSADRDA criteria [29]. Exclusion criteria were: prominent frontal lobe symptoms, clinical or brain imaging signs of cerebrovascular disease, family history of dementia, treatment with lithium, warfarin, memantine, antidepressants, and neuroleptics. MMSE was performed at baseline to select only mild to moderate severity (MMSE score $>15$ ) AD cases. Treatment with AChE inhibitors at stable doses had to be ongoing for at least 3 months prior to the study. Within 4 weeks after enrolment, subjects underwent cognitive tests 
MMSE and Alzheimer's disease Assessment Scale, Cognitive subscale (ADAS-Cog), and CSF tapping. Six months later, cognitive tests were repeated and a second CSF sample was taken. All patients gave informed consent to participate in the study. The study was conducted according to the Helsinki Declaration and approved by the ethics committee at the University of Lund and Uppsala and at the Karolinska Institute, Stockholm, Sweden.

\section{Isolation of NDEV and PDEV}

EVs were isolated from human serum following a protocol previously described, with some alterations [31]. Briefly, $0.5 \mathrm{~mL}$ of serum from four AD CSF biomarker positive and four AD CSF biomarker negative subjects were collected and frozen. Cut-offs used to for a biomarker positive CSF profile were A $\beta 1-42<550 \mathrm{ng} / \mathrm{L}$, t-tau $>400 \mathrm{ng} / \mathrm{L}$, and p-tau $>80 \mathrm{ng} / \mathrm{L}$. After thawing, $500 \mu \mathrm{l}$ of Dulbecco's solution were added to the samples. Samples were mixed, left for $5 \mathrm{~min}$ at RT, and then centrifuged at $4000 \times \mathrm{g}$ for $20 \mathrm{~min}$ at $4{ }^{\circ} \mathrm{C}$. Supernatants were transferred to new tubes and mixed with $252 \mu$ l Exoquick ${ }^{\circledR}$ exosome precipitating solution (System Biosciences). Samples were incubated for $60 \mathrm{~min}$ at $4{ }^{\circ} \mathrm{C}$ and then centrifuged at $1500 \mathrm{~g}$ for $20 \mathrm{~min}$ at $4{ }^{\circ} \mathrm{C}$. Supernatants were discarded after centrifugation and the pellet was re-suspended in $0.5 \mathrm{ml} \mathrm{dH}_{2} \mathrm{O}$. Samples were incubated for $1 \mathrm{~h}$ at $4{ }^{\circ} \mathrm{C}$ with $4 \mu \mathrm{g}$ in $50 \mu \mathrm{L}$ of $3 \%$ BSA of mouse antihuman CD171 biotinylated antibody (eBio5G3) directed to neuronal $\mathrm{L} 1$ cell adhesion molecule (L1CAM). Next, $15 \mu \mathrm{L}$ of streptavidin-agarose Ultralink resin (Thermo Scientific) was added with $25 \mu \mathrm{L}$ of $3 \%$ BSA and incubated for $30 \mathrm{~min}$ at $4{ }^{\circ} \mathrm{C}$ with continuous mixing. Samples were centrifuged at $200 \mathrm{~g}$ for $10 \mathrm{~min}$ at $4{ }^{\circ} \mathrm{C}$ and the supernatants were saved for the analysis of PDEV, while the pellet was re-suspended in $200 \mu \mathrm{L}$ of $0.1 \mathrm{M}$ glycine $\mathrm{HCl}$ by mixing for $10 \mathrm{~s}$ and centrifuged at $4500 \mathrm{~g}$ for $10 \mathrm{~min}$ at $4{ }^{\circ} \mathrm{C}$ to detach L1CAM + EVs from the beads. Supernatants were transferred to new tubes and $25 \mu \mathrm{L} \mathrm{3 \%} \mathrm{BSA} \mathrm{and} 15 \mu \mathrm{L}$ of $1 \mathrm{M}$ Tris $-\mathrm{HCl}$ was added. Exosomes were lysed by addition of $260 \mu \mathrm{L}$ mammalian protein extraction reagent (M-PER; Thermo Scientific) and went through two freeze thaw cycles. Samples were stored at $-80{ }^{\circ} \mathrm{C}$ pending immunoassay analysis.

\section{Statistical analysis}

GraphPad Prism v7.02 (GraphPad Software, Inc., La Jolla, CA, USA) was used for the statistical analysis of discovery, validation, longitudinal, and PSP/CBS cohorts. Data were not normally distributed, so the Mann-Whitney and Kruskal-Wallis tests were used for the comparison of the groups. The significant threshold was set to $p<0.05$. Correlation was tested used the Spearman rank correlation coefficient. Although non-parametric tests were used, linear regression lines were added to the figures as guidance. SPSS v25.0 (IBM Corp., Armonk, N.Y., USA) was used to fit the generalized linear model in the longitudinal cohort. Baseline MMSE values (intercept) and rate of change (slope) were used as outcomes in linear regression models, with CSF $\mathrm{N}-123$ and N-224 tau as predictors. Subjects were divided into quartile groups according to CSF N-123 and N-224 levels at baseline and linear regression models between the quartile groups were compared. In the biomarker stability study, statistical analysis was carried out using the R programming language (version 3.4.3), and all tests were twosided with a significance threshold set to $p<0.05$. There were no corrections made for multiple comparisons, so any $p$ values close to 0.05 should be considered exploratory. Change in tau N-224 across treatments was tested with non-parametric Kruskal-Wallis test. Post hoc analysis of differences in biomarker concentration between baseline and follow-up and between specific groups was carried out with the Mann-Whitney $U$ test. Differences in biomarker concentration across varying number of copies of the $\varepsilon 4$ allele of the $A P O E$ gene were also tested in this way. Differences in biomarker concentration across treatments depending on $A P O E \& 4$ genotypes were tested by fitting a linear model with an interaction term between treatment and $\varepsilon 4$ copies, while both age and sex were included as covariates. The Spearman rank correlation coefficient was used to assess the relationship between change in biomarker concentration and age, sex, and change in treatment outcomes as measured by the MMSE and ADAS-Cog scales.

\section{Results}

\section{Semi-tryptic tau peptides reveal endogenous cleavage sites}

Tau was enriched from both brain tissue homogenates and CSF using immunoprecipitation (IP) with Tau12 and treated with trypsin. In brain tissue, tryptic peptides could be detected spanning the whole tau protein, i.e., from the $\mathrm{N}$-terminus (acetylated aa 2) to the C-terminus (aa 441). Peptides representing all isoforms $(0 \mathrm{~N}, 1 \mathrm{~N}, 2 \mathrm{~N}, 3 \mathrm{R}$, and 4R) were detected. In CSF, only peptides representing the $\mathrm{N}$-terminal half of tau were detected; the most C-terminal peptide ended at aa 254 and isoform-specific tryptic peptides originating from $0 \mathrm{~N}, 1 \mathrm{~N}$, and $2 \mathrm{~N}$ tau isoforms were detected (Fig. 2, Supplementary Table 1). Peptides phosphorylated at threonine 181 were detected both in brain tissue and in CSF. Furthermore, in both brain and CSF, a number of semi-tryptic peptides, i.e. peptides with either the $\mathrm{N}$ - or $\mathrm{C}$-terminus generated by cleavage by trypsin and the other end by endogenous proteases, were identified. Among these were peptides ending at aa 123, 124, and 
Fig. 2 Top: endogenous tau species found with $\mathrm{N}$-terminal and mid-region-specific antibodies (Tau 12 in red, HT7 in blue, BT2 in orange) and neo-epitope-specific antibodies (Tau_C123 in lime, Tau_C224 in green) with selected amino acid positions indicated. Alignment and numbering refer to the $2 \mathrm{~N}$ isoform; for peptides originating from the $0 \mathrm{~N}$ and $1 \mathrm{~N}$ isoforms, $2 \mathrm{~N}$ sequence portions not included are dimmed. The protein schematic structure shows the respective antibody epitopes and T181. Bottom: in black, range (Ac-2-254) of tryptic peptides detected in CSF with Tau12 (regions with no coverage are dimmed). Vertical lines indicate possible tryptic cleavage sites

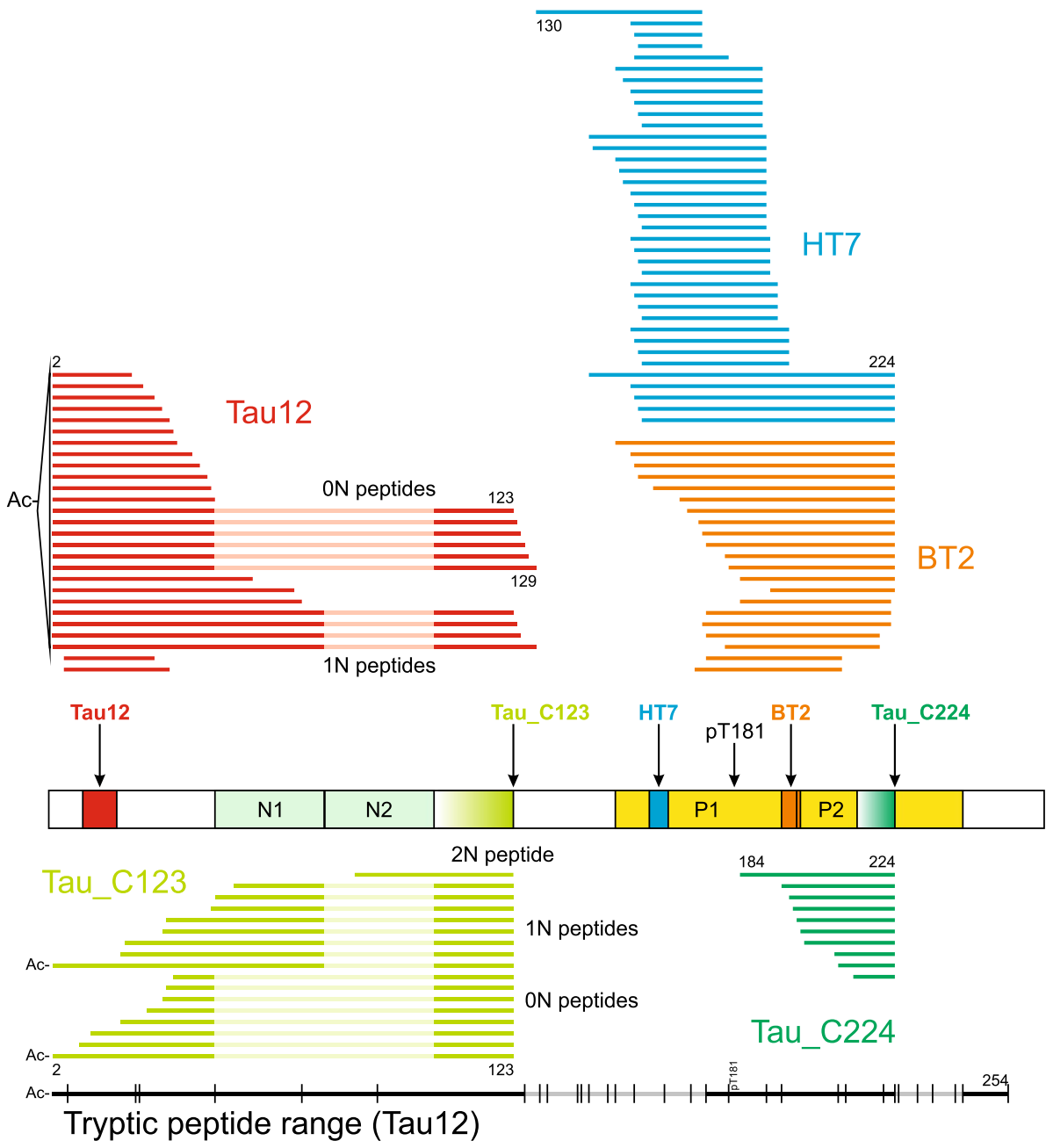

125, which led us to investigate non-trypsinated samples containing endogenous tau fragments in CSF. We also investigated which tau species were immunoprecipitated when using antibodies with different binding epitopes on tau (Supplementary Table 2). Quantitative data were obtained by spiking a stable-isotope-labeled tau 1N4R protein into the CSF prior to IP. When performing IP with Tau12, tryptic peptides were abundant in the region close to the antibody epitope, while intensities decreased when moving in the C-terminal direction (Fig. 3). For HT7 and BT2, a similar situation was observed; the abundance was high close to the epitopes but decreased markedly at the C-terminal side, but not as clearly toward the N-terminus (Fig. 3). Together, these data indicate that there is an abundance of shorter tau species present in CSF, both at the N-terminus and in the mid-region around aa 150-200. There are also longer species, but it appears that, shortly after aa 221, the abundance drops significantly, and C-terminally of aa 254 no tryptic peptides were detected when using these three antibodies (Fig. 3).

\section{Endogenous tau fragments ending at aa 123 and 224 are prominent in CSF}

Analyses of endogenous tau peptides were performed without tryptic digestion, to preserve the naturally occurring peptides in CSF. Using IP-MS with Tau12, 27 different endogenous tau fragments were identified by MS/MS (excluding oxidized variants at the three available methionine, Met). The majority of these peptides contained the $\mathrm{N}$-terminus of tau protein. The N-terminal peptides were lacking the Met1 amino acid, and were acetylated at the $\mathrm{N}$-terminus. Endogenous species from both the $0 \mathrm{~N}$ and $1 \mathrm{~N}$ isoforms were observed, ranging up to aa 129 and including peptides ending C-terminally at aa 123 (Fig. 2 and Supplementary Table 3). With HT7 and BT2, 37 and 21 different mid-region species were identified, respectively, ranging from aa 130 to aa 224. With HT7, which recovers both T181 and pT181 forms, additional peptides phosphorylated at T181 were observed. From the detected peptides from the tau mid-region, it was notable that a major cleavage site was situated between aa 224 and aa 
Fig. 3 Representation of quantitative MS data of tau fragments immunoprecipitated with Tau 12, HT7, and BT2

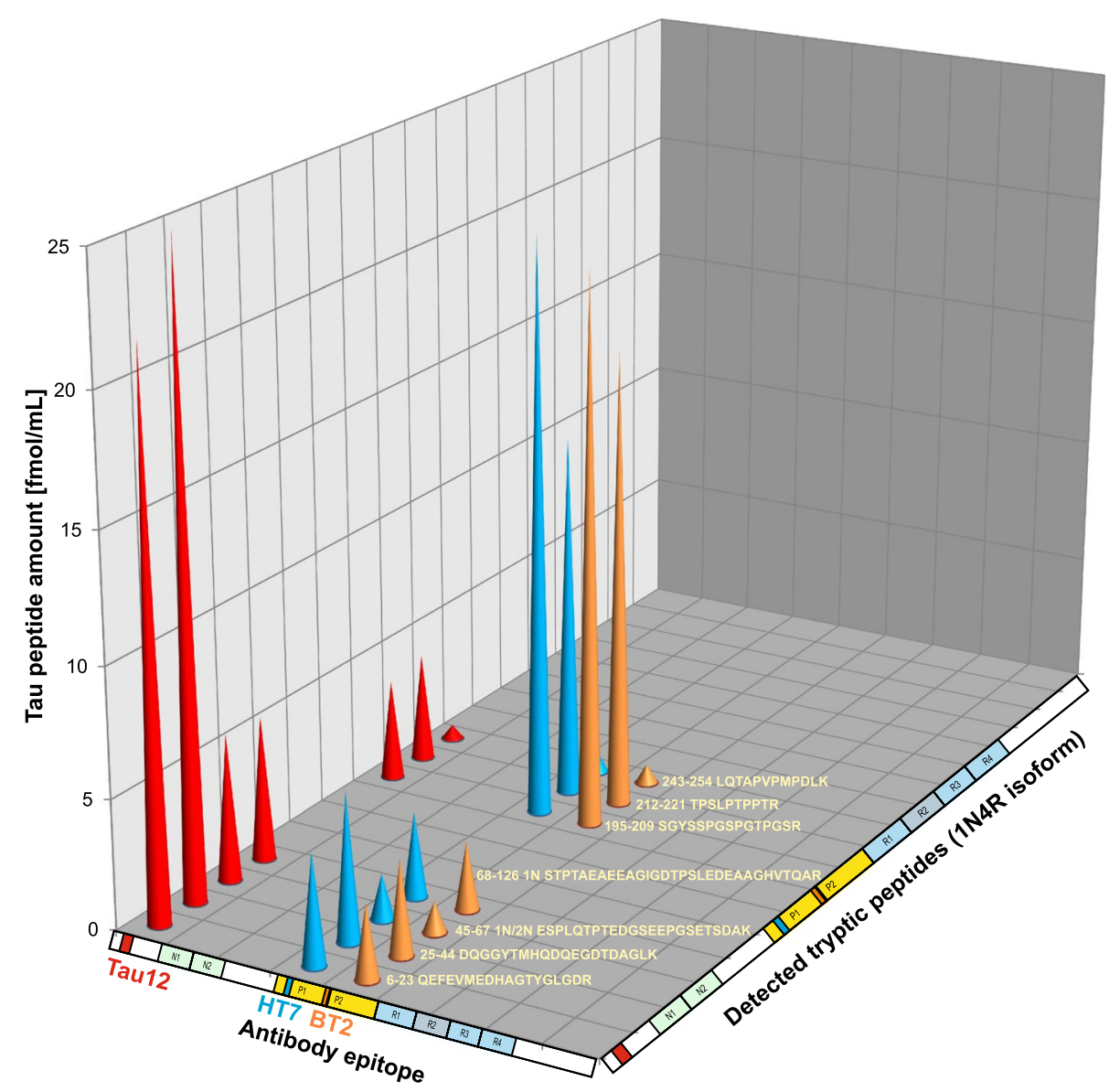

225. The top portion of Fig. 2 shows a summary of the endogenous tau species identified.

\section{Newly developed neo-epitope antibodies are specific against aa 123 and aa 224}

A total of 17 tau species, all ending C-terminally at aa 123 and belonging to all three isoforms ( $0 \mathrm{~N}, 1 \mathrm{~N}$, and $2 \mathrm{~N})$, were identified when performing IP-MS of CSF using the anti-tau 123 clone "Tau_1". Supplementary Fig. 1a shows the MS/MS data for the tau peptide Ac-2-63... 103-123 from the $1 \mathrm{~N}$ isoform (the gap between 63 and 103 is because aa $64-102$ are present only in the $2 \mathrm{~N}$ isoform). Similarly, when performing IP-MS of CSF using the anti-tau 224 clone "Tau_F", ten different tau species ending C-terminally at aa 224 were detected. Supplementary Fig. 1b shows the MS/MS data for the tau peptide 197-224. The bottom portion of Fig. 2 and Supplementary Table 3 shows a summary of the endogenous tau species detected when using these two antibodies.

\section{Anti-224 antibody (but not anti-123) stains neurofibrillary tangles}

Immunohistochemical analysis with anti-tau 224 antibody demonstrated neurofibrillary tangles and dystrophic neurites surrounded by neuropil threads, whilst faint punctate neuronal cytoplasmic staining was observed in the normal control (Fig. 4a-c). The anti-tau 123 antibody showed weak neuronal cytoplasmic staining in $\mathrm{AD}$ which was negative in the normal control (Fig. 4d-f). Immunohistochemical staining on sequential sections with anti-AT8 shows the presence of neurofibrillary tangles in $\mathrm{AD}$ and dystrophic neurites (Fig. 4g) and the filamentous structure of the neurofibrillary tangles (Fig. 4h). No AT8 staining was present in the normal control (Fig. 4i).

\section{$\mathrm{N}-224$ tau and t-tau concentrations show a decreasing trend in AD brain samples along Braak stages}

There was no significant difference in the concentration between $\mathrm{AD}$ and controls for the $\mathrm{N}-123$ fragment $(p=0.5)$ 
Fig. 4 Tau immunohistochemistry in Alzheimer's disease ( $\mathbf{a}$ and $\mathbf{b} ; \mathbf{d}$ and $\mathbf{e} ; \mathbf{g}$ and $\mathbf{h}$ ) and a neurologically normal control (c, f, and i). Anti-tau 224 immunohistochemistry shows neurofibrillary tangles (a, arrow) and dystrophic neurites (a, double arrow) surrounded by neuropil threads. Neurofibrillary tangles show at higher magnification (b). Faint punctuate neuronal cytoplasmic staining was observed in the normal control. Neurons in $\mathrm{AD}$ are weakly positive for anti-tau 123 and negative in the normal control. For comparison, sequential sections were immunohistochemically stained with AT8 to show the presence of neurofibrillary tangles in AD (g, arrow), dystrophic neurites (g, double arrow), and filamentous structure of the neurofibrillary tangles (h). AT8 staining is absent in the normal control. Bar (in i) represents $50 \mu \mathrm{m}$ in $\mathbf{a}, \mathbf{c}, \mathbf{d}, \mathbf{f}, \mathbf{g}$, and $\mathbf{i} ; 20 \mu \mathrm{m}$ in $\mathbf{b}$, $\mathbf{e}$, and $\mathbf{h}$

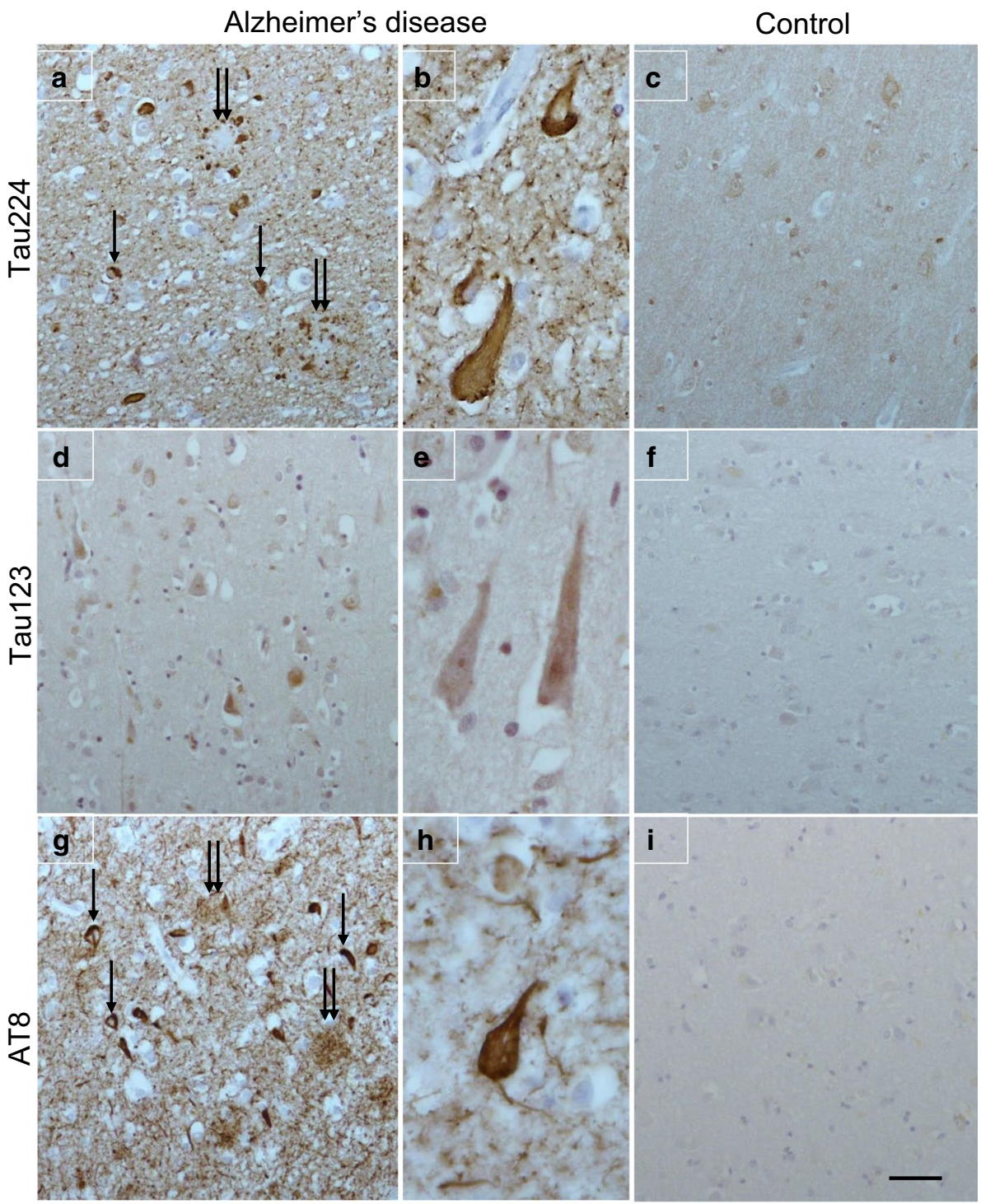

(Fig. 5a). The N-224 tau fragment was present in AD brain at significantly lower concentrations compared to controls $(p=0.01)$, although not significant when normalized to total protein content $(p=0.06)$ (Fig. 5b). T-tau measurement shows significantly lower concentrations in AD compared to controls both for absolute and normalized concentrations ( $p=0.01$ and $p=0.004$, respectively) (Fig. 5c). When dividing the samples by Braak staging, the N-224 fragment and t-tau tended to decrease from stage I to VI when including all samples and in AD samples from stage III-VI (Fig. 5e). No visible trend is present for the fragment N-123 (Fig. 5d) or for any of the tau fragments in controls.

\section{$\mathrm{N}-123$ and $\mathrm{N}-224$ tau concentrations in CSF are significantly increased in AD in a discovery cohort}

The CSF concentration of tau N-123 was significantly higher in AD compared to non-AD subjects $(p<0.001)$ (Supplementary Fig. 2a). There was a significant positive correlation between the $\mathrm{N}-123$ fragment and t-tau in the AD group $(r=0.56, p=0.01)$ but not in the control group $(r=0.15$, $p=0.58)$. Similarly, the concentration of the tau N-224 was significantly higher in AD compared to controls $(p<0.001)$ (Supplementary Fig. 2b). Furthermore, a significant positive correlation was found between tau N-224 tau and t-tau in the 
Fig. 5 Brain levels (normalized for total protein content) of $\mathrm{N}-123$ (a), N-224 (b) fragments, and t-tau (c) in Alzheimer's disease and controls and in AD over Braak stages III-VI $(\mathbf{d}, \mathbf{e}, \mathbf{f})$ (lines representing the median, bars representing interquartile range)
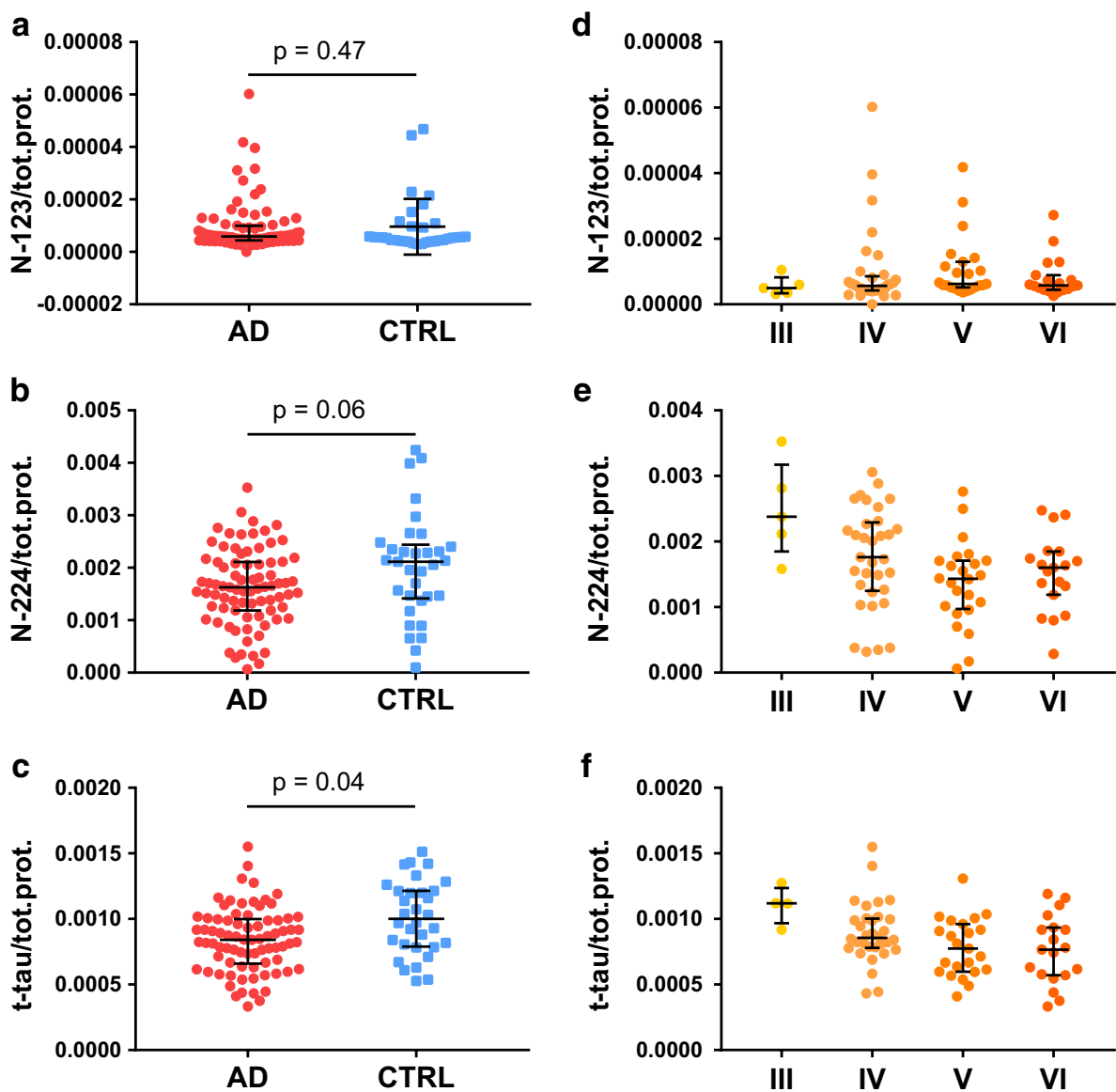

control group $(r=0.65, p=0.004, \alpha<0.05)$ but not in the AD group $(r=0.432, p=0.1308)$. N-123 and N-224 showed a positive correlation in $\mathrm{AD}(r=0.9, p<0.0001)$ but not in controls. A significant correlation was also found between $\mathrm{N}-224$ tau and N-mid domain tau (N-mid, aa 9-198) in AD $(r=0.72, p=0.01)$ as well as in controls $(r=0.69, p=0.01)$.

\section{$\mathrm{N}-224$ and $\mathrm{x}-224$ tau (but not $\mathrm{N}-123)$ concentrations in CSF are significantly increased in AD in a validation cohort}

For tau N-123, we observed an opposite behaviour compared to the discovery cohort, with significantly higher concentrations in controls vs. AD ( $p=0.001)$ (Fig. 6a). N-123 concentrations did not correlate with t-tau (Fig. 6d). In contrast, tau N-224 was significantly higher in patients with AD compared to controls $(p<0.001)$ (Fig. 6b). Tau N-224 correlated to t-tau in both $\mathrm{AD}$ and controls ( $r=0.62$ vs. $r=0.75$ ) and the correlation was significant in both groups $(p<0.0001)$ (Fig. 6e). N-123 and N-224 had a positive correlation in controls $(r=0.5, p<0.0001)$ but not in AD. N-224 also shows a significant correlation to N-mid domain tau (N-mid, aa 9-198), ( $r=0.81$ and $r=0.76$ for AD and controls, respectively, $p<0.0001$ for both). Tau $\mathrm{x}-224$ concentration was significantly higher in AD CSF samples compared to controls $(p<0.0001)$ (Fig. 6c). Tau x-224 correlates positively to t-tau in $\mathrm{AD}$ and control cohorts ( $r=0.8$ and 0.9 , respectively) and both correlations are significant $(p<0.0001)$ (Fig. 6f).

\section{$\mathrm{N}-123$ and $\mathrm{N}-224$ tau baseline concentrations in CSF are related to severity of cognitive impairment in a longitudinal cohort}

Baseline levels of the N-123 and N-224 fragments were significantly higher in AD vs. OND ( $p=0.03$ and 0.002 , respectively), mild cognitive impairment due to AD (MCI-AD) vs. MCI ( $p=0.01 ; p=0.002)$, and MCI-AD vs. OND $(p=0.001$; $p<0.0001$ ) (Fig. 7a, b). Group differences for N-123 and $\mathrm{N}-224$ did not change significantly when controlling for age and gender effects $(p>0.05)$. N-123 and N-224 showed a positive correlation in MCI $(r=0.59, p=0.001)$, MCI-AD $(r=0.61, p=0.004)$, and OND group $(r=0.67, p=0.005)$, but not in AD. Based on the baseline CSF N-123 and N-224 tau levels, AD, MCI-AD and MCI groups were classified into quartiles (Q1, Q2, Q3, and Q4), which were tested for association with disease progression as examined by longitudinal change in Mini-Mental State Examination (MMSE). 
Fig. 6 Validation cohort. Left column: concentration range of $\mathrm{N}-123(\mathbf{a}), \mathrm{N}-224(\mathbf{b})$, and $\mathrm{x}-224$ (c) fragments in $\mathrm{AD}$ and controls (lines representing median; bars representing interquartile range; in a, bars removed due to the LOG scale). Right column: correlation of $\mathrm{N}-123(\mathbf{d}), \mathrm{N}-224$ (e), and x-224 (f) fragment to $\mathrm{t}$-tau and linear regression in $\mathrm{AD}$ and controls
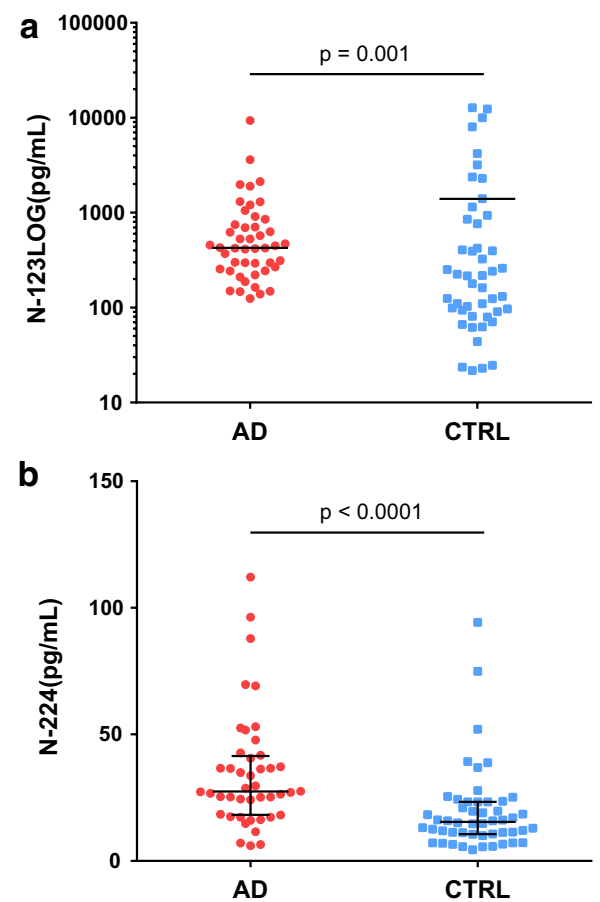

e

d
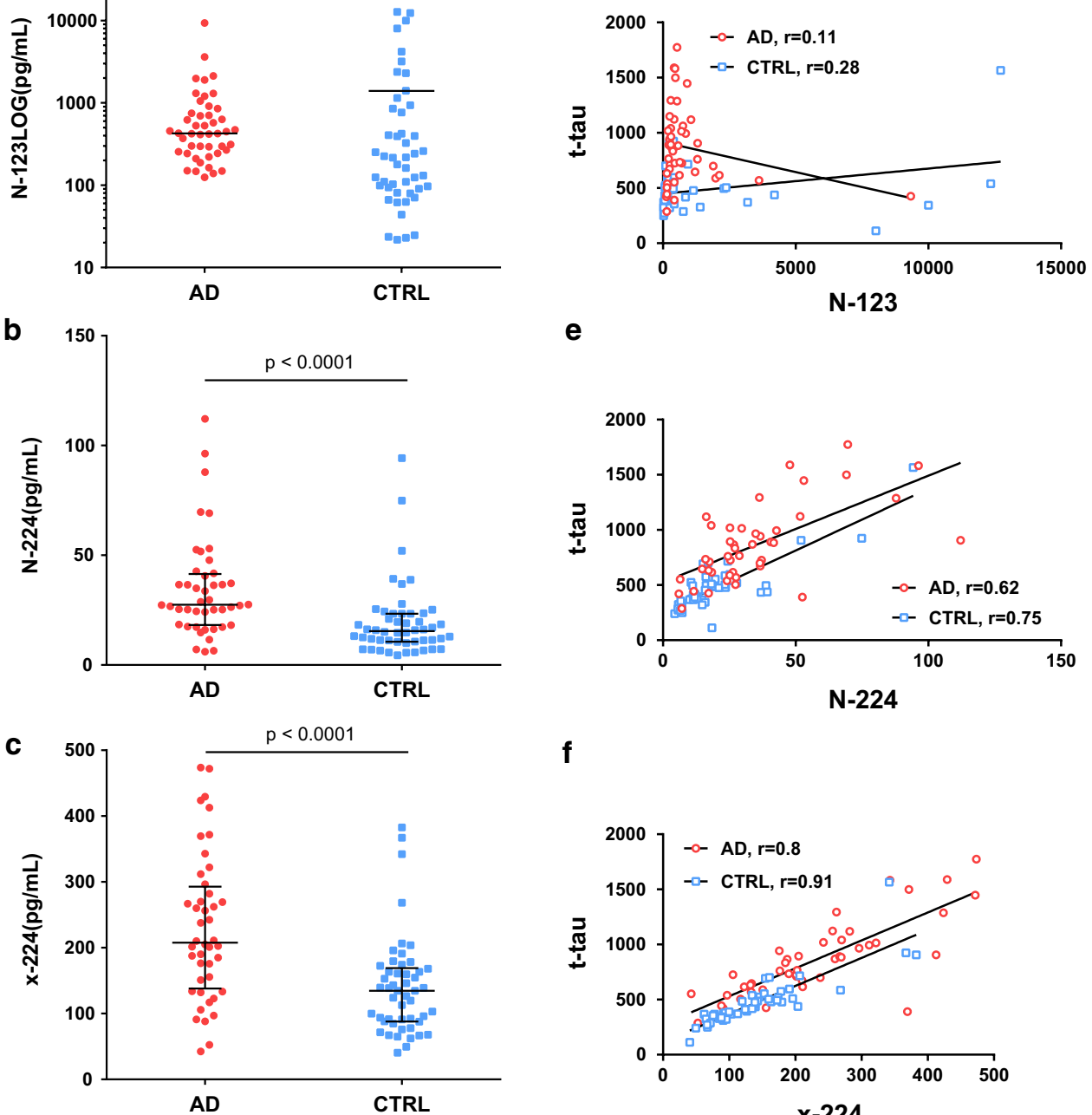

f

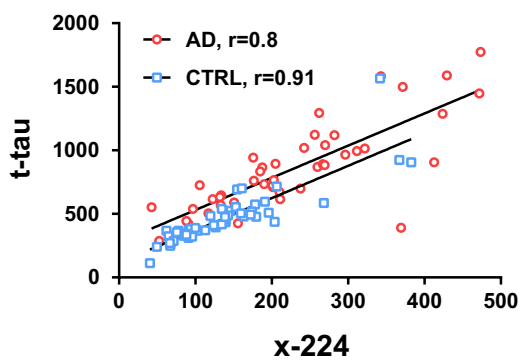

MMSE score decreased significantly over years from baseline for the highest quartile (Q4) for both fragments $(\mathrm{N}-123$ : adj. $R^{2}=0.36, \beta=-0.61, p=0.0001 ; \mathrm{N}-224$ : adj. $R^{2}=0.34$, $\beta=-0.58, p=0.0002$ ) (Fig. 7c, d). A significant decrease at MMSE score in Q4 was not observed for t-tau and p-tau $(p>0.05)$.

\section{$\mathrm{N}-224$ tau does not correlate to $t$-tau and $\mathrm{p}$-tau in PSP and CBS}

CSF levels of N-224 tau were significantly lower in PSP and CBS vs. both AD ( $p<0.0001$, both) and controls (PSP: $p<0.0001$; CBS: $p<0.002$ ) (Fig. 8a). CSF N-224 tau concentrations did not significantly correlate to t-tau in both PSP ( $r=0.09)$ and CBS ( $r=0.33)$ (Fig. 8b). Correlation to p-tau was significant in PSP $(r=0.54, p=0.002)$ but not in CBS ( $r=0.33$ ) (Fig. 8c). After removing the A $\beta+$ subjects, no significant correlation with t-tau or $\mathrm{p}$-tau was present in any of the groups (PSP: $r=0.48$ for both: CBS: $r=0.39, r=0.11$ ) (Fig. 8e-f). Concentrations of N-224 tau in A $\beta$ - PSP were significantly lower than in AD and controls $(p<0.0001)$, while, in CBS, concentrations were significantly lower only when compared to $\operatorname{AD}(p=0.0001)$ (Fig. 8d). N-123 tau concentrations were below LLOQ in 8 out of $15(53.3 \%)$ CBS cases and 19 out of $31(61.3 \%)$ PSP cases.

\section{N-224 tau concentrations in CSF are not affected by treatment with $\mathrm{AChE}$ inhibitors}

Levels of tau N-224 did not significantly differ between baseline and follow-up ( $p=0.78)$, with an average $\mathrm{CV}$ of $13.4 \%$ and $12 \%$ change (Supplementary Fig. 3a, b, c). The concentrations were not significantly different across drug treatments $(p=0.08)$. There were no significant differences between specific groups when comparing donepezil to rivastigmine treatment $(p=0.28)$ and donepezil to galantamine $(p=0.37)$. Moreover, N-224 tau levels did not differ 
Fig. 7 Longitudinal cohort. a, b Concentration range of $\mathrm{N}-123$ (a) and $\mathrm{N}-224$ (b) fragments in $\mathrm{AD}, \mathrm{MCI}$, and MCI-AD and OND (lines representing the median, bars representing interquartile range). c, $\mathbf{d}$ Disease progression over time in $\mathrm{AD}$, MCI-AD and MCI cohorts, as measured by change in MMSE scores. Higher levels of CSF $\mathrm{N}-123$ (a) and N-224 (b) tau at baseline are related to faster decline. N-123: Quartile (Q) 1: $n=16, \mathrm{Q} 2: n=15, \mathrm{Q} 3: n=15$ and Q4: $n=16$; N-224: Q1: $n=17, \mathrm{Q} 2: n=17, \mathrm{Q} 3: n=17$, and Q4: $n=17$
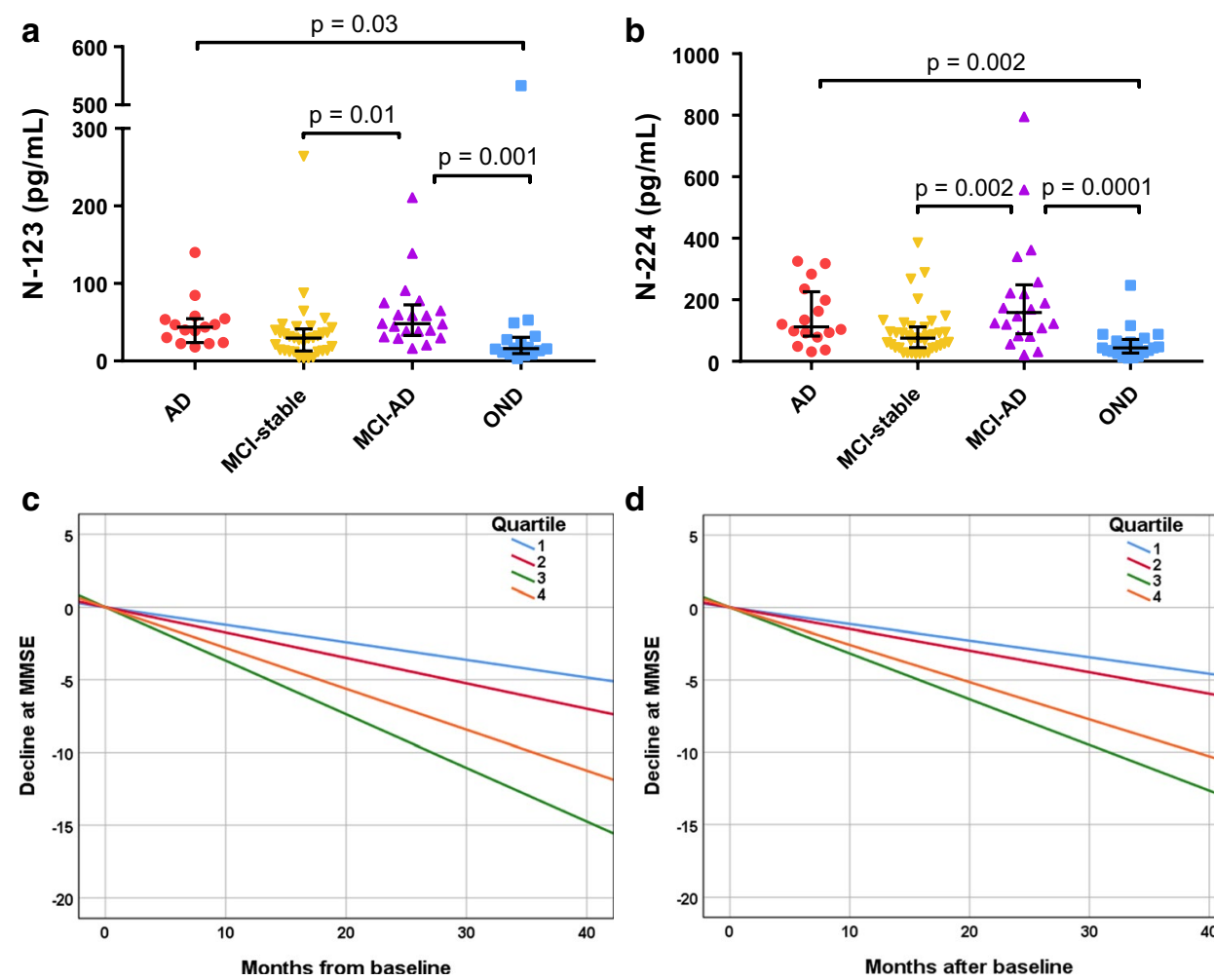

d

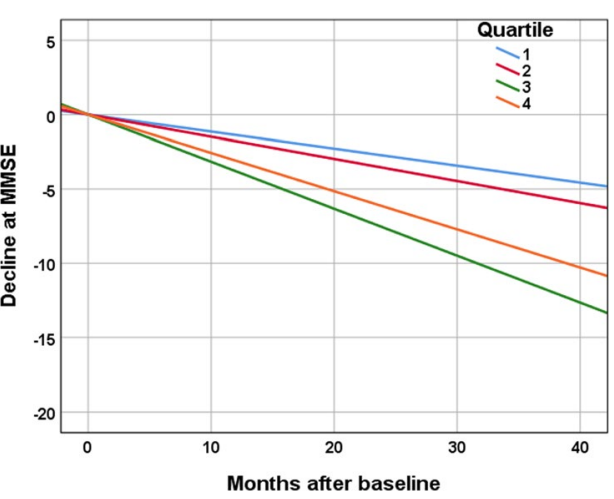

a
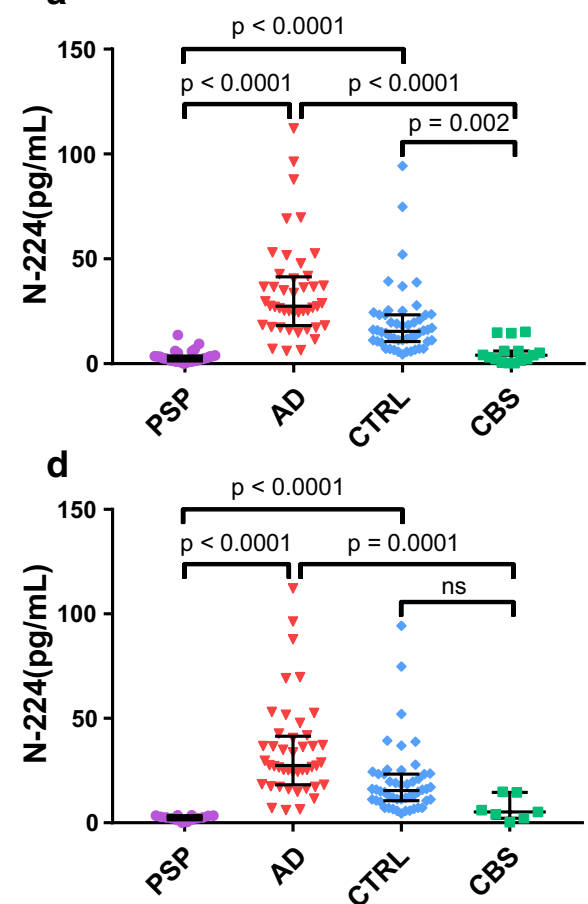

b

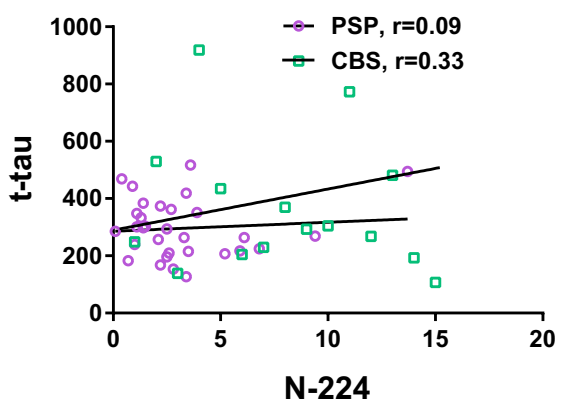

e

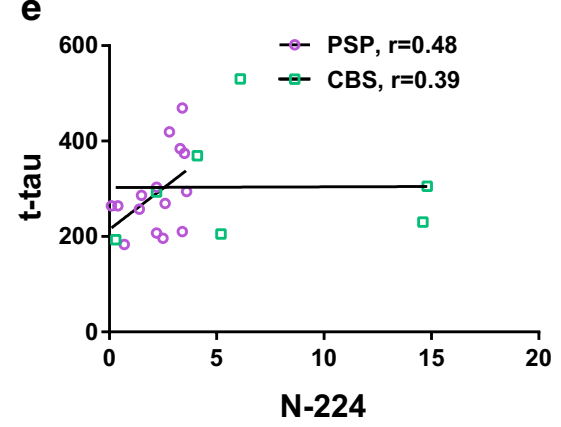

c

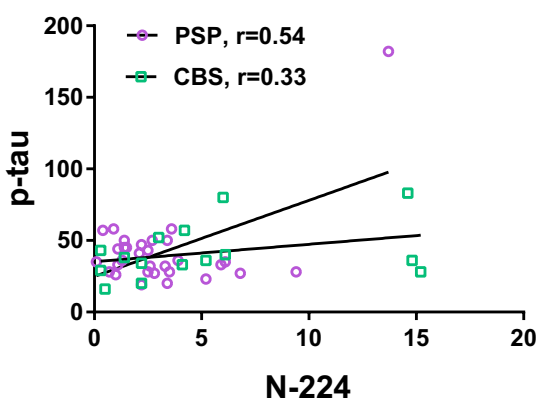

f

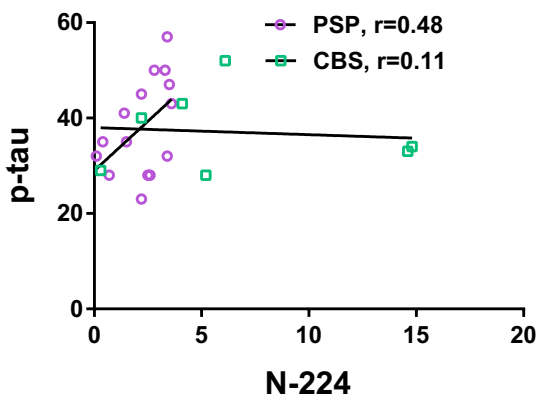

Fig. 8 PSP and CBS cohort. a, $\mathbf{d}$ Concentration of N-224 tau in PSP and CBS (a: $\mathrm{A} \beta+$ and $\mathrm{A} \beta$; $\mathbf{d}$ : $\mathrm{A} \beta$ - only) compared to $\mathrm{AD}$ and controls from the validation cohort (lines representing the median, bars representing interquartile range). b, c, e, f Correlation to t-tau (b) and p-tau (c) and linear regression in PSP and CBS cohorts (b, c: A $\beta+$ and $\mathrm{A} \beta ; \mathrm{e}, \mathrm{f}: \mathrm{A} \beta-$ only) 
significantly depending on number of $A P O E \varepsilon 4$ copies $(p=0.48)$. There was no interaction effect between treatment and APOE $\varepsilon 4$ copies for tau N-224 ( $p=0.35)$. There was no strong association between change in tau N-224 and either change in MMSE $(r=-0.12, p=0.38)$ or change in ADAS-Cog $(r=0.03, p=0.81)$. Tau N-224 did not differ across sex $(p=0.22)$, or age $(p=0.08)$.

\section{$\mathrm{N}-224$ tau specifically present in NDEV and N-123 tau in both NDEV and PDEV}

The N-123 tau fragment was measurable in all EV lysates with no significant difference between NDEVs, i.e., L1CAM-positive samples, and peripherally derived EVs (PDEVs), i.e., supernatants left after L1CAM-positive
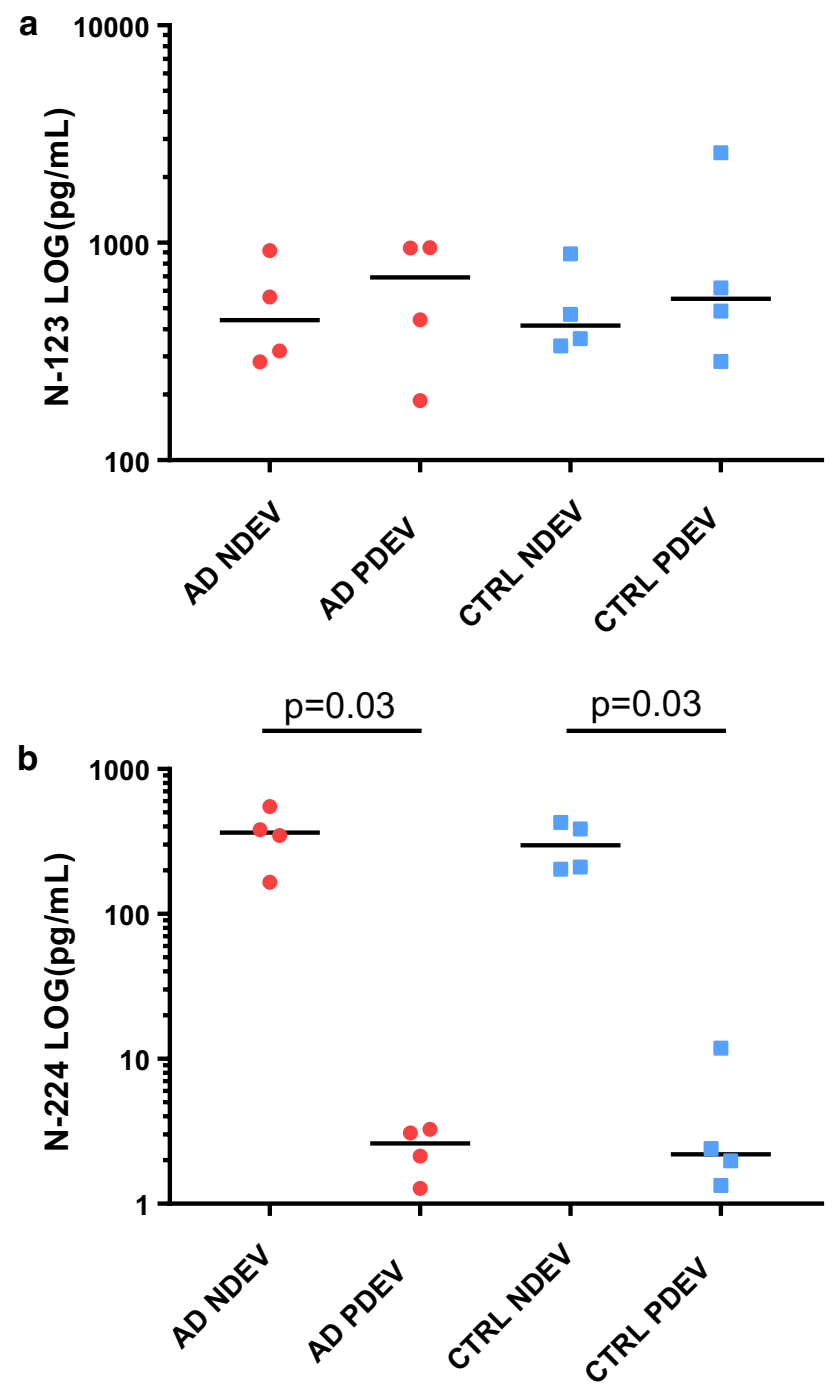

Fig. 9 Scatter dot plots of N-123 (a) and N-224 tau (b) concentrations in serum extracellular vesicles (EVs) lysates, divided in AD and control neuronally derived EVs (NDEV) and peripherally derived EVs (PDEV) samples (lines representing median)
EVs had been bound to the beads. There were no differences comparing levels of tau N-123 in NDEVs or PDEVs comparing $\mathrm{AD}$ and control samples. Comparable concentrations of the fragments were observed within and between cohorts (Fig. 9a). Conversely, the N-224 tau fragment was significantly higher in NDEV lysates compared to PDEV lysates $(p=0.03)$ in both AD and control groups. Fold differences between average concentrations of N-224 tau in NDEV samples compared to PDEV were 148 and 70 in the $\mathrm{AD}$ and control group, respectively. Differences between $\mathrm{AD}$ and control NDEV samples were not significant (Fig. 9b).

\section{Discussion}

Using IP-MS analysis with anti-tau antibodies (Tau 12, HT7, and BT2), we collected quantitative data demonstrating several tau fragments in CSF, both at the $\mathrm{N}$-terminus and in the mid-region (Fig. 2). Shortly after aa 221, the abundance of the fragment intensities dropped significantly and C-terminally of aa 254 no tryptic peptides were detected when using these three antibodies (Fig. 3). The two major tau pools detected were composed of peptides ending at aa 123 and aa 224, respectively, suggesting prominent proteolytic cleavage of the protein around these sites. It should be noted that the absence of even longer peptides corresponding to the data from trypsinated CSF samples is likely a methodological bias; MS favors detection and identification of smaller peptides and there are likely longer tau variants present in CSF which evade identification with the LC-MS configuration used in the current study. Furthermore, the choice of a C18 column for separation may also have contributed to the bias against longer peptides.

In support of the findings in the present study, the previous studies in brain tissue identified N-terminally truncated fragments starting from aa 124 and proteomics studies on CSF confirmed the presence of fragments ending at aa 224 $[9,18,35]$. We, therefore, generated antibodies against these specific fragment epitopes and confirmed their specificity through ELISA cross-reactivity tests as well as IP-MS. The antibodies were successfully tested for immunohistochemistry on AD and control brain slices, and used to develop targeted immunoassays. Validation of immunoassays on different platforms (ELISA, Simoa), and their application on exosome lysates, soluble fraction of brain extracts and CSF from four independent cohorts of AD and control subjects, showed that the novel assays were specific, sensitive, and robust, with fragment concentrations within the calibration range.

Immunohistochemical staining showed positivity to the anti-tau 224 antibody in neurofibrillary tangles and neuropil threads (Fig. 4a, b), suggesting that this fragment is deposited in $\mathrm{AD}$ and colocalized with the tangles. Data from brain 
extracts showed an apparent decreasing trend for the $\mathrm{N}-224$ tau fragment over the course of disease (Braak III-VI), with lower concentration in AD brain compared to control, although the difference was not statistically significant when normalized to total protein content (Fig. 5b, e). T-tau shows the same behaviour in brain extracts, being significantly lower in $\mathrm{AD}$ brain compared to controls, even when normalized to total protein content (Fig. 5c, f). Although it might appear counterintuitive, this behaviour is justified by the technique used for extraction: RIPA buffer extracts all of the fractions soluble in weak detergents and also a proportion of tau insoluble in no-detergent or non-ionic detergent conditions, but not tau aggregated into tangles, as it would require additional extraction steps. These findings suggest that $\mathrm{N}-224$ might be trapped in the fuzzy coat of tau tangles and, therefore, less likely to be extracted with this technique in samples from later stages of disease, although these observation need to be confirmed with further experiments, i.e., sarkosyl extraction and treatment of the sarkosyl-insoluble pellet. On the other hand, in CSF the N-224 tau fragment shows an opposite behaviour to the brain, being significantly higher in AD CSF compared to control and in MCI-AD compared to stable MCI (Figs. 6b, 7b). Thus, brain and CSF give us complementary information about tau metabolism in AD compared to controls.

It seems, therefore, that, in the CNS, there is a diseaserelated upregulated cleavage at this site which leads to enhanced release from neurons of the N-terminal fragment, ultimately leading to higher CSF concentration of the fragment and a deposition into tangles. At a tangle level, the 224-cleaved tau might, therefore, be an indirect marker of ongoing tangle formation. Another potential explanation for the results (with similar practical implications regarding the possible association of the fragment with tangle pathology) is that the production of the fragment could be enhanced in the fuzzy coat of tangles. These promising results open up for the investigation of tau fragments in tangle pathology, using various techniques, such as laser capture of tangles and more refined extraction techniques.

Several studies have been published on N-terminal cleavage of tau from proteases of the calpain and caspase family [20, 25]. The N-224 might also represent the neurotoxic $\mathrm{N}$-terminal tau fragment of 20-22 $\mathrm{kDa}$ that has been shown by western blot in several studies $[1-4,8]$. Other studies have also shown the presence of $\mathrm{N}$-terminal fragments ranging from 20 to $40 \mathrm{kDa}$ in CSF [30]. Secretion of N-terminal tau fragments has also been previously described [36]. Further studies to establish which enzyme is responsible for this cleavage and whether this activity is specifically upregulated in $\mathrm{AD}$ are needed.

The fragment $\mathrm{x}-224$ proved to be less interesting than $\mathrm{N}-224$, meaning that it more tightly correlated with t-tau in $\mathrm{AD}$ and controls, probably due to the overlapping region of interest of the assays used to measure the two tau entities (aa 159-240). Tau N-224, on the other hand, might represent the product of a different metabolic route affecting the $\mathrm{N}$-terminus of the protein as opposed to the mid-region pool.

When analyzing baseline CSF values of N-224 tau in subjects monitored over time by means of MMSE, we observed that the magnitude of cognitive decline is related to the initial concentration of the fragment (Fig. 7d). It seems, therefore, that not only the N-224 fragment is AD-related, but its concentration could also have a predictive value of worse cognitive outcome. In the previous studies, no correlation was found between baseline core CSF biomarker levels and follow-up cognitive scores [39]. One limitation of our study is that the subjects were monitored by MMSE, which is not the optimal test to detect the early AD-related changes in cognition [40]. Future studies on prognostic value of tau fragments should be performed in longitudinal cohorts where cognitive assessment at follow-up includes ADASCog and delayed word recall [34].

When comparing AD to primary tauopathies PSP and CBS, we observe that CSF N-224 tau concentrations are significantly decreased in PSP and CBS, being even lower than in controls. We have also measured core AD CSF biomarkers to discriminate AD-like PSP and CBS. Even after excluding CSF $A \beta+$ subjects, the same behaviour is observed, with the loss of significant difference between CBS and controls possibly due to a statistical power issue (seven CBS cases vs. 50 controls). A similar behaviour in PSP and CBS has been observed before for t-tau and p-tau [21, 41, 42]. This might be due to an overall lower concentration of CSF tau in the primary tauopathies, and it is not known whether this is due to reduced tau production, reduced secretion into the CSF, or both. Difference in the distribution of tau pathology between neurons (AD) and glia (PSP and CBD) might also account for this behaviour. The lack of correlation of N-224 tau to $\mathrm{p}$-tau and $\mathrm{t}$-tau suggests a different underlying disease mechanism compared to AD, which does not include cleavage of N-terminal tau. Parallel investigation of N-224 tau, t-tau, and p-tau in PSP and CBS could help to understand these different mechanisms and add to the information from classic CSF tau biomarkers.

In terms of stability and predictive power, it is reassuring that the levels of N-224 tau in AD subjects were basically unaffected by $\mathrm{AChE}$ inhibitor treatment at 6-month followup, regardless of $A P O E$ status, age, gender, or drug type. This type of study would benefit from a longer follow-up, to evaluate possible long-term oscillations in the concentration and association with cognitive scores.

Immunohistochemical data on $\mathrm{N}-123$ tau showed only a faint positivity of neuronal staining with anti-tau 123 and mostly in the cytoplasm, which makes the presence of $\mathrm{N}-123$ tau in the tangles unlikely and reinforces the idea of an active secretion of the fragment (Fig. 4d-f). A similar 
behaviour for the N-123 fragment was observed in CSF and brain extracts, with a wide range of concentrations in both AD and control cohorts (Figs. 5a, 6a). More precisely, the difference in concentration between the two groups is not consistent throughout the cohorts, and no trend is visible over the course of disease as measured by Braak staging. Although the N-123 fragment does not seem to have a clear diagnostic role, the previous publications suggest that the function of tau is affected by $\mathrm{N}$-terminal shedding and that $\mathrm{N}$-terminal integrity is necessary for microtubule stability, tau solubility, and cell survival [5, 18, 24]. One possible speculation is that this tau fragment might represent a general marker for tau metabolism occurring in CNS and peripheral nervous system, a hypothesis reinforced by its presence in the PDEV. However, no indications have been found on how this relates to the disease state and/or stage, although higher CSF levels at baseline are related to higher rate of cognitive decline at follow-up as measured by decline at MMSE (Fig. 7c). Data from serum EVs do not corroborate the brain or disease specificity of the fragment, showing similar level of N-123 in NDEV and PDEV from AD and controls (Fig. 9a). These findings are opposed to the ones on $\mathrm{N}-224$ tau in serum EV lysate; here, N-224 appears to be brain-specific, showing concentrations close to zero in PDEV, although not being decisive in discriminating between AD and controls (Fig. 9b). It is possible that there is an intrinsic balance between tau fragmentation and stability, and that the $\mathrm{N}-123$ fragment could be a marker of physiological processes within a given range of concentrations. It is also evident that N-123 is more abundant tau fragment than $\mathrm{N}-224$, being readily measureable by ELISA, which is much less sensitive than Simoa. Moreover, no correlation between the concentrations of $\mathrm{N}-123$ and $\mathrm{N}-224$ tau is present in the $\mathrm{AD}$ groups of the validation and longitudinal cohorts, while it is present in control, OND, MCI, and MCI-AD groups. The biological mechanism underlying the difference in abundance between N-123 and N-224 is currently unknown, but the aggregated results suggest that the two fragments might reflect different mechanism of tau processing in AD.

In conclusion, our data suggest that, among the several fragments of tau present in CSF, there are two major pools consisting of tau species cleaved at either aa 123 or 224 . While cleavage generating tau N-123 could be part of the normal function of tau turnover, the generation of tau N-224 is related to AD. In support of this hypothesis is the lack of consistent differences of CSF N-123 tau levels comparing $\mathrm{AD}$ and control, while fragments ending at aa 224 are significantly higher in AD CSF compared to control and relate to the decrease in cognitive performance over time. Future studies evaluating whether these tau cleavages may promote tau aggregation and propagation are of high interest. Furthermore, studies in primary tauopathies show great promise for elucidating the role of differential tau processing in $\mathrm{AD}$ compared to the other tauopathies.

Acknowledgements Dr. Thomas McAvoy (Merck Research Laboratories) kindly provided the stable-isotope labeled tau 1NR4 protein used for quantitative MS studies. Maria Olsson (University of Gothenburg) produced the recombinant Tau 123 and Tau 224 proteins.

Author contributions CC performed the experiments, developed and validated the assays, analyzed the data, and wrote the manuscript. GB performed the MS analysis. JW optimized the exosome extraction, and provided the exosome lysates. EP and JG participated in the study design and gave technical assistance. $\mathrm{OH}, \mathrm{LP}$, and RC selected the clinical cohorts and provided CSF samples. KW, HHC, and TGB collected and provided the brain samples. TL performed the immunohistochemistry. $\mathrm{NC}$ supervised and participated in the statistical analysis. $\mathrm{HZ}, \mathrm{KB}$, and $\mathrm{KH}$ designed the study. All authors have reviewed the manuscript.

Funding This study was funded by grants from the Sweden and European Research Council, the Torsten Söderberg Foundation at the Royal Swedish Academy of Sciences, the Knut and Alice Wallenberg Foundation, Frimurarestiftelsen, Stiftelsen för Gamla Tjänarinnor, Agrens foundation, the Swedish Alzheimer's Foundation, and the Swedish Brain Foundation. We are grateful to the Banner Sun Health Research Institute Brain and Body Donation Program of Sun City, Arizona for the provision of post-mortem human cerebral cortex. The Brain and Body Donation Program has been supported by the National Institute of Neurological Disorders and Stroke (U24 NS072026 National Brain and Tissue Resource for Parkinson's Disease and Related Disorders), the National Institute on Aging (P30 AG19610 Arizona AD Core Center), the Arizona Department of Health Services (contract 211,002, Arizona Alzheimer's Research Center), the Arizona Biomedical Research Commission (contracts 4001, 0011, 05-901 and 1001 to the Arizona Parkinson's Disease Consortium), and the Michael J. Fox Foundation for Parkinson's Research. The Queen Square Brain Bank is supported by the Reta Lila Weston Institute for Neurological Studies and the Progressive Supranuclear Palsy (Europe) Association. TL is supported by an Alzheimer's Research UK senior fellowship.

Conflict of interest $\mathrm{OH}$ has acquired research support (for the institution) from Roche, GE Healthcare, Biogen, AVID Radiopharmaceuticals, Fujirebio, and Euroimmun. In the past 2 years, he has received consultancy/speaker fees (paid to the institution) from Lilly, Roche, and Fujirebio. KW and HHC are employed at Genentech, South San Francisco, CA, USA. HZ has served at scientific advisory board meetings for Roche Diagnostics, Wave, Samumed, and CogRx, and is a co-founder of Brain Biomarker Solutions in Gothenburg AB, a GU Venture-based platform company at the University of Gothenburg. LP has served at scientific advisory board meetings for Fujirebio, IBL and Roche.

Open Access This article is distributed under the terms of the Creative Commons Attribution 4.0 International License (http://creativeco mmons.org/licenses/by/4.0/), which permits unrestricted use, distribution, and reproduction in any medium, provided you give appropriate credit to the original author(s) and the source, provide a link to the Creative Commons license, and indicate if changes were made. 


\section{References}

1. Amadoro G, Corsetti V, Atlante A, Florenzano F, Capsoni S, Bussani R et al (2012) Interaction between NH(2)-tau fragment and Abeta in Alzheimer's disease mitochondria contributes to the synaptic deterioration. Neurobiol Aging 33(833):833-e1. https:// doi.org/10.1016/j.neurobiolaging.2011.08.001

2. Amadoro G, Corsetti V, Florenzano F, Atlante A, Ciotti MT, Mongiardi MP et al (2014) AD-linked, toxic NH2 human tau affects the quality control of mitochondria in neurons. Neurobiol Dis 62:489-507. https://doi.org/10.1016/j.nbd.2013.10.018

3. Amadoro G, Corsetti V, Sancesario GM, Lubrano A, Melchiorri G, Bernardini S et al (2014) Cerebrospinal fluid levels of a 20-22 kDa NH2 fragment of human tau provide a novel neuronal injury biomarker in Alzheimer's disease and other dementias. J Alzheimer's Dis 42:211-226. https://doi.org/10.3233/ JAD-140267

4. Amadoro G, Corsetti V, Stringaro A, Colone M, D'Aguanno S, Meli G et al (2010) A NH2 tau fragment targets neuronal mitochondria at AD synapses: possible implications for neurodegeneration. J Alzheimer's Dis 21:445-470. https://doi.org/10.3233/ JAD-2010-100120

5. Amadoro G, Serafino AL, Barbato C, Ciotti MT, Sacco A, Calissano P et al (2004) Role of N-terminal tau domain integrity on the survival of cerebellar granule neurons. Cell Death Differ 11:217-230. https://doi.org/10.1038/sj.cdd.4401314

6. Andreasen N, Hesse C, Davidsson P, Minthon L, Wallin A, Winblad B et al (1999) Cerebrospinal fluid beta-amyloid(1-42) in Alzheimer disease: differences between early- and late-onset Alzheimer disease and stability during the course of disease. Arch Neurol 56:673-680

7. Armstrong MJ, Litvan I, Lang AE, Bak TH, Bhatia KP, Borroni B et al (2013) Criteria for the diagnosis of corticobasal degeneration. Neurology 80:496-503. https://doi.org/10.1212/ WNL.0b013e31827fofd 1

8. Atlante A, Amadoro G, Bobba A, de Bari L, Corsetti V, Pappalardo $\mathrm{G}$ et al (2008) A peptide containing residues 26-44 of tau protein impairs mitochondrial oxidative phosphorylation acting at the level of the adenine nucleotide translocator. Biochim Biophys Acta 1777:1289-1300. https://doi.org/10.1016/j.bbabi o.2008.07.004

9. Barthelemy NR, Fenaille F, Hirtz C, Sergeant N, SchraenMaschke S, Vialaret J et al (2016) Tau protein quantification in human cerebrospinal fluid by targeted mass spectrometry at high sequence coverage provides insights into its primary structure heterogeneity. J Proteome Res 15:667-676. https:// doi.org/10.1021/acs.jproteome.5b01001

10. Barthelemy NR, Gabelle A, Hirtz C, Fenaille F, Sergeant N, Schraen-Maschke $S$ et al (2016) Differential mass spectrometry profiles of tau protein in the cerebrospinal fluid of patients with Alzheimer's disease, progressive supranuclear palsy, and dementia with lewy bodies. J Alzheimer's Dis 51:1033-1043. https://doi.org/10.3233/JAD-150962

11. Beach TG, Adler CH, Sue LI, Serrano G, Shill HA, Walker DG et al (2015) Arizona study of aging and neurodegenerative disorders and brain and body donation program. Neuropathology 35:354-389. https://doi.org/10.1111/neup.12189

12. Blennow K, Hampel H, Weiner M, Zetterberg H (2010) Cerebrospinal fluid and plasma biomarkers in Alzheimer disease. Nat Rev Neurol 6:131-144. https://doi.org/10.1038/nrneurol.2010.4

13. Blennow K, Wallin A, Agren H, Spenger C, Siegfried J, Vanmechelen E (1995) Tau protein in cerebrospinal fluid: a biochemical marker for axonal degeneration in Alzheimer disease? Mol Chem Neuropathol 26:231-245. https://doi.org/10.1007/BF028 15140
14. Blennow K, Zetterberg H, Minthon L, Lannfelt L, Strid S, Annas $P$ et al (2007) Longitudinal stability of CSF biomarkers in Alzheimer's disease. Neurosci Lett 419:18-22. https://doi.org/10.1016/j. neulet.2007.03.064

15. Borroni B, Premi E, Agosti C, Alberici A, Cerini C, Archetti S et al (2011) CSF Alzheimer's disease-like pattern in corticobasal syndrome: evidence for a distinct disorder. J Neurol Neurosurg Psychiatry 82:834-838. https://doi.org/10.1136/jnnp.2010.221853

16. Braak H, Braak E (1991) Neuropathological stageing of Alzheimer-related changes. Acta Neuropathol 82:239-259. https://doi. org/10.1007/bf00308809

17. Brinkmalm G, Portelius E, Ohrfelt A, Mattsson N, Persson R, Gustavsson MK et al (2012) An online nano-LC-ESI-FTICR-MS method for comprehensive characterization of endogenous fragments from amyloid beta and amyloid precursor protein in human and cat cerebrospinal fluid. J Mass Spectrom 47:591-603. https:// doi.org/10.1002/jms.2987

18. Derisbourg M, Leghay C, Chiappetta G, Fernandez-Gomez FJ, Laurent C, Demeyer D et al (2015) Role of the tau N-terminal region in microtubule stabilization revealed by new endogenous truncated forms. Sci Rep 5:9659. https://doi.org/10.1038/srep0 9659

19. Dubois B, Feldman HH, Jacova C, Hampel H, Molinuevo JL, Blennow K et al (2014) Advancing research diagnostic criteria for Alzheimer's disease: the IWG-2 criteria. Lancet Neurol 13:614 629. https://doi.org/10.1016/S1474-4422(14)70090-0

20. Garg S, Timm T, Mandelkow EM, Mandelkow E, Wang Y (2011) Cleavage of tau by calpain in Alzheimer's disease: the quest for the toxic $17 \mathrm{kD}$ fragment. Neurobiol Aging 32:1-14. https://doi. org/10.1016/j.neurobiolaging.2010.09.008

21. Hall S, Ohrfelt A, Constantinescu R, Andreasson U, Surova Y, Bostrom F et al (2012) Accuracy of a panel of 5 cerebrospinal fluid biomarkers in the differential diagnosis of patients with dementia and/or parkinsonian disorders. Arch Neurol 69:14451452. https://doi.org/10.1001/archneurol.2012.1654

22. Hansson KT, Skillback T, Pernevik E, Kern S, Portelius E, Hoglund $\mathrm{K}$ et al (2017) Expanding the cerebrospinal fluid endopeptidome. Proteomics 17(5):1600384. https://doi.org/10.1002/ pmic. 201600384

23. Hoglund K, Bogstedt A, Fabre S, Aziz A, Annas P, Basun H et al (2012) Longitudinal stability evaluation of biomarkers and their correlation in cerebrospinal fluid and plasma from patients with Alzheimer's disease. J Alzheimer's Dis 32:939-947. https://doi. org/10.3233/JAD-2012-120976

24. Horowitz PM, LaPointe N, Guillozet-Bongaarts AL, Berry RW, Binder LI (2006) N-terminal fragments of tau inhibit full-length tau polymerization in vitro. Biochemistry 45:12859-12866. https ://doi.org/10.1021/bi061325g

25. Horowitz PM, Patterson KR, Guillozet-Bongaarts AL, Reynolds MR, Carroll CA, Weintraub ST, Bennett DA, Cryns VL, Berry RW, Binder LI (2004) Early N-terminal changes and caspase-6 cleavage of tau in Alzheimer's disease. J Neurosci 24:7895-7902. https://doi.org/10.1523/JNEUROSCI.1988-04.2004

26. Lashley T, Rohrer JD, Bandopadhyay R, Fry C, Ahmed Z, Isaacs AM et al (2011) A comparative clinical, pathological, biochemical and genetic study of fused in sarcoma proteinopathies. Brain 134:2548-2564. https://doi.org/10.1093/brain/awr160

27. Litvan I, Bhatia KP, Burn DJ, Goetz CG, Lang AE, McKeith I et al (2003) Movement disorders society scientific issues committee report: SIC task force appraisal of clinical diagnostic criteria for Parkinsonian disorders. Mov Disord 18:467-486. https://doi. org/10.1002/mds. 10459

28. McKhann G, Drachman D, Folstein M, Katzman R, Price D, Stadlan EM (1984) Clinical diagnosis of Alzheimer's disease: report of the NINCDS-ADRDA work group under the auspices 
of department of health and human services task force on Alzheimer's disease. Neurology 34:939-944

29. McKhann GM, Knopman DS, Chertkow H, Hyman BT, Jack CR Jr, Kawas CH et al (2011) The diagnosis of dementia due to Alzheimer's disease: recommendations from the national institute on aging-Alzheimer's Association workgroups on diagnostic guidelines for Alzheimer's disease. Alzheimer's Dement 7:263-269. https://doi.org/10.1016/j.jalz.2011.03.005

30. Meredith JE Jr, Sankaranarayanan S, Guss V, Lanzetti AJ, Berisha F, Neely RJ et al (2013) Characterization of novel CSF Tau and ptau biomarkers for Alzheimer's disease. PLoS One 8:e76523. https://doi.org/10.1371/journal.pone.0076523

31. Mustapic M, Eitan E, Werner JK Jr, Berkowitz ST, Lazaropoulos MP, Tran J et al (2017) Plasma extracellular vesicles enriched for neuronal origin: a potential window into brain pathologic processes. Fron Neurosci 11:278. https://doi.org/10.3389/fnins.2017.00278

32. Olsson B, Lautner R, Andreasson U, Ohrfelt A, Portelius E, Bjerke $\mathrm{M}$ et al (2016) CSF and blood biomarkers for the diagnosis of Alzheimer's disease: a systematic review and meta-analysis. Lancet Neurol 15:673-684. https://doi.org/10.1016/S1474 -4422(16)00070-3

33. Petersen RC (2004) Mild cognitive impairment as a diagnostic entity. J Intern Med 256:183-194. https://doi.org/10.111 1/j.1365-2796.2004.01388.x

34. Rosen WG, Mohs RC, Davis KL (1984) A new rating scale for Alzheimer's disease. Am J Psychiatry 141:1356-1364. https://doi. org/10.1176/ajp.141.11.1356

35. Russell CL, Mitra V, Hansson K, Blennow K, Gobom J, Zetterberg $\mathrm{H}$ et al (2017) Comprehensive quantitative profiling of tau and phosphorylated tau peptides in cerebrospinal fluid by mass spectrometry provides new biomarker candidates. J Alzheimer's Dis 55:303-313. https://doi.org/10.3233/JAD-160633

36. Sato C, Barthelemy NR, Mawuenyega KG, Patterson BW, Gordon BA, Jockel-Balsarotti J et al (2018) Tau kinetics in neurons and the human central nervous system. Neuron 97(1284-1298):e1287. https://doi.org/10.1016/j.neuron.2018.02.015

37. Scheltens P, Blennow K, Breteler MM, de Strooper B, Frisoni GB, Salloway S et al (2016) Alzheimer's disease. Lancet (Lond Engl) 388:505-517. https://doi.org/10.1016/S0140-6736(15)01124-1

38. Sjogren M, Davidsson P, Tullberg M, Minthon L, Wallin A, Wikkelso C et al (2001) Both total and phosphorylated tau are increased in Alzheimer's disease. J Neurol Neurosurg Psychiatry 70:624-630

39. Stomrud E, Hansson O, Zetterberg H, Blennow K, Minthon L, Londos E (2010) Correlation of longitudinal cerebrospinal fluid biomarkers with cognitive decline in healthy older adults. Arch Neurol 67:217-223. https://doi.org/10.1001/archneurol.2009.316

40. Tombaugh TN (2005) Test-retest reliable coefficients and 5-year change scores for the MMSE and 3MS. Arch Clin Neuropsychol 20:485-503. https://doi.org/10.1016/j.acn.2004.11.004

41. Urakami K, Wada K, Arai H, Sasaki H, Kanai M, Shoji M et al (2001) Diagnostic significance of tau protein in cerebrospinal fluid from patients with corticobasal degeneration or progressive supranuclear palsy. J Neurol Sci 183:95-98

42. Wagshal D, Sankaranarayanan S, Guss V, Hall T, Berisha F, Lobach I et al (2015) Divergent CSF tau alterations in two common tauopathies: Alzheimer's disease and progressive supranuclear palsy. J Neurol Neurosurg Psychiatry 86:244-250. https:// doi.org/10.1136/jnnp-2014-308004

43. Vanmechelen E, Vanderstichele H, Davidsson P, Van Kerschaver E, Van Der Perre B, Sjogren M et al (2000) Quantification of tau phosphorylated at threonine 181 in human cerebrospinal fluid: a sandwich ELISA with a synthetic phosphopeptide for standardization. Neurosci Lett 285:49-52

44. World Health Organization and Alzheimer's Disease International (2012) Dementia: a public health priority. ISBN: 978924 1564458

\section{Affiliations}

\section{Claudia Cicognola ${ }^{1}$ (D) Gunnar Brinkmalm ${ }^{1}$. Jessica Wahlgren ${ }^{1}$. Erik Portelius ${ }^{1,2}$. Johan Gobom ${ }^{1,2}$. Nicholas C. Cullen ${ }^{3}$. Oskar Hansson ${ }^{4,5} \cdot$ Lucilla Parnetti $^{6} \cdot$ Radu Constantinescu $^{7} \cdot$ Kristin Wildsmith $^{8}$. Hsu-Hsin Chen ${ }^{9}$. Thomas G. Beach ${ }^{10} \cdot$ Tammaryn Lashley $^{11}$ • Henrik Zetterberg ${ }^{1,2,12,13}$. Kaj Blennow ${ }^{1,2}$. Kina Höglund ${ }^{1,2,14}$}

1 Institute of Neuroscience and Physiology, Department of Psychiatry and Neurochemistry, The Sahlgrenska Academy at University of Gothenburg, Göteborgsvägen 31, House V3/SU, 43180 Mölndal, Sweden

2 Clinical Neurochemistry Laboratory, Sahlgrenska University Hospital, Mölndal, Sweden

3 Department of Neurology, Perelman School of Medicine, University of Pennsylvania, Philadelphia, PA, USA

4 Clinical Memory Research Unit, Department of Clinical Sciences Malmö, Lund University, Lund, Sweden

5 Memory Clinic, Skåne University Hospital, Malmö, Sweden

6 Department of Medicine, Center for Memory Disturbances, Laboratory of Clinical Neurochemistry, Neurology Clinic, University of Perugia, Santa Maria della Misericordia Hospital, Perugia, Italy

7 Institute of Neuroscience and Physiology, Department of Neurology, The Sahlgrenska Academy at University of Gothenburg, Gothenburg, Sweden
8 Biomarker Development Department, Genentech, South San Francisco, CA, USA

9 Biomarker Discovery Department, Genentech, South San Francisco, CA, USA

10 Civin Laboratory for Neuropathology, Banner Sun Health Research Institute, Sun City, AZ, USA

11 Queen Square Brain Bank for Neurological Disorders, Department of Movement Disorders, Institute of Neurology, University College London, London, UK

12 Department of Neurodegenerative Disease, UCL Institute of Neurology Queen Square, London, UK

13 UK Dementia Research Institute at UCL, London, UK

14 Department of Neurobiology, Care Sciences and Society, Center for Alzheimer Research, Division for Neurogeriatrics, Karolinska Institutet, Novum, Huddinge, Stockholm, Sweden 